\title{
Synthesis, in-silico Designing, SAR and Microbiological Evaluation of Novel Amide Derivatives of 1-(4-Nitrophenyl)-2-(3-methylbenzo[b] thiophen-6-yl)-1H-benzo[d]imidazole-5-carboxylic Acid
}

\author{
Tanuj Hooda ${ }^{1,2,}$, Sunil Sharma ${ }^{3}$, Naveen Goyal ${ }^{4}$ \\ ${ }^{1}$ Uttarakhand Technical University, Dehradun, Uttarakhand, INDIA. \\ ${ }^{2}$ Faculty of Pharmaceutical Chemistry Vaish Institute of Pharmaceutical Education and Research, Rohtak, Haryana, INDIA. \\ ${ }^{3}$ Department of Pharmaceutical Sciences, Guru Jambheshwar University of Science and Technology, Hissar, Haryana, INDIA. \\ ${ }^{4}$ Himachal Institute of Pharmacy, Paonta Sahib, Himachal Pradesh, INDIA.
}

\begin{abstract}
Background: Due to this increasing predicament of antibiotic confrontation, the lot of distinct antibiotics available is dwindling and there are only smatterings of new antibiotics in the drug development channel. Therefore, an intense necessitate for new antimicrobial drugs. In current study, we have synthesized the new derivatives with the help of molecular docking studies and investigated antimicrobial activities. Methods: By focusing the enzymes i.e. aminoacyl-tRNA synthetase (AaRS) and tyrosyl-tRNA synthetase, new amides of 2-(3-methylbenzo[b]thiophen-6-yl)-1-(4-nitrophenyl)-1H-benzo[d]imidazole-5-carboxylic acid were synthesized and inhibition by docking study precedence to antimicrobial action. Studies were accomplished on a designed library of amide derivatives with the help of docking softwares i.e. AutoDock Vina 4.2 and Schrodinger's maestro package against crystal structure of enzymes (PDB ID: 1 wny.PDBOT and1jil.PDBOT). Based upon their dock score, superlative 23 focused amide derivatives of 2-(3-methylbenzo[b]thiophen-6-yl)1-(4-nitrophenyl)-1H-benzo[d]imidazole-5-carboxylic acid were synthesized and further investigated for in vitro antimicrobial. Results: Among the library, superlative compounds C-1, C-10 and C-19 having tremendous dock score by AutoDock Vina/Schrodinger's maestro against 1 wny.PDBQT:1jil.PDBQT-9.0:-9.9/ -5.144:-5.328,-9.2:-9.4/ -5.007:5.369, -9.0:-9.6/ -5.184:-5.264 as compared to standard drug dock score -10.2:10.6/-5.847:-5.895.C-1, C-10 and C-19 derivatives also exhibited good MIC against six representative micro-organisms as compared to standard by tube dilution method. Conclusion: In-vitro results revealed that a large number of synthesized compounds having good antimicrobial activity and dock score can be used for in vivo studies for future. In-silico studies and in vitro results comply with each other.

Key words: Benzimidazole, 1jil.PDBQT, 1 wny.PDBQT, Antimicrobial Activity, SAR.
\end{abstract}

\section{INTRODUCTION}

At present lot of drugs are accessible for curing the microbial infection but most of them is becoming abortive due to antimicrobial resistance by the micro-organisms. So there is an immense need for the discovery of novel antimicrobial agents to overcome the antimicrobial resistance and side effects. ${ }^{1}$ The tyrosyl-tRNA synthetase and
aminoacyl-tRNA synthetase (AaRS) enzymes have been a hub of latest research for drug discovery and play decisive roles in biosynthesis of protein by catalyzing the synthesis aminoacylation of transfer RNA (tRNA) by their cognate amino acid. Once enzymes are inhibited, halted protein biosynthesis, which in turn outcome in the shrinking
Submission Date: 20-09-2019; Revision Date: 19-11-2019; Accepted Date: 07-01-2020

DOI: 10.5530/ijper.54.2.54 Correspondence: Mr. Tanuj Hooda, Uttarakhand Technical University, Dehradun, Uttarakhand, INDIA Assistant Professor, Vaish Institute of Pharmaceutical Education and Research, 1274 Sector-1,

Rohtak-124001, Haryana, INDIA.

Phone: +91 8950342053 E-mail: tanujhooda2010@ gmail.com

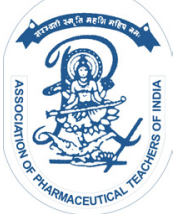

www.ijper.org 
of bacterial growth under both in vitro and contagious conditions. ${ }^{2,3}$ Docking softwares are extremely valuable for the screening of abundance of molecules affinity towards a selective disease or drug target. ${ }^{4}$

The chemistry and pharmacology of benzimidazole have been purposeful to medicinal chemistry because benzimidazole derivatives possessed various pharmacological activities i.e. antimicrobial, antioxidant, anticancer, anti-inflammatory, analgesic, antidiabetic, anticonvulsant, anti-asthmatic, antiviral, antihypertensive, antiproliferative, antitubercular with many more. ${ }^{5-14}$ Benzimidazole moiety have been showing talented/ flourishing activity in the healing of numerous diseases and due to that reason benzimidazoles attained much contemplation as meaningful pharmacophore and called as honored structure in medicinal chemistry. ${ }^{15}$ By examine review contains the various benzimidazoles and their pharmacological, focused amide derivatives of 2-(3-methylbenzo[b]thiophen-6-yl)-1-(4-nitrophenyl)$1 \mathrm{H}$-benzo[d]imidazole-5-carboxylic acid were taken for lead development depending upon their docking score. In last decade, the virtual screening approach for studying the docking of small moieties with protein structure is a authoritative tool for lead design and has become the most active and valuable technology now being used in drug discovery processing. It is an effort to remove the element of fortune from the drug designs, because pharmacological activity is reliant upon drug receptor binding which was analyzed by molecular docking. ${ }^{16}$ In the present study, we designed the focused amide derivatives of 2-(3-methylbenzo[b]thiophen-6-yl)-1(4-nitrophenyl)-1H-benzo[d]imidazole-5-carboxylic acid were tested for docking to study the binding modes and binding affinities to the 1wny. PDBQT (Isolencyl-tRNA synthetase editing domain, source: Thermus thermophilus) and 1jil.PDBQT(tyrosyl-tRNA synthetase editing domain, source: Staphylococcus aureus) in which aminoacyl-tRNA synthetase (AaRS) and tyrosyl-tRNA synthetase enzymeshave been a center of attention of recent research for bacterial inhibitory activity by using software AutoDock Vina 4.2 and Schrodinger Glide. ${ }^{17-19}$ Both software's showed exceptional non-commercial researcher docking program that is broadly used around the world. The final screening of the focused amide derivatives of 2-(3-methylbenzo[b]thiophen-6-yl)1-(4-nitrophenyl)-1H-benzo[d]imidazole-5-carboxylic acidis calculated. Molecular docking helps in studying the interaction between the receptor/protein with ligand by identifying the suitable active/binding sites in protein and by obtaining the finest geometry of ligand-receptor complex and calculating the energy of interaction between ligands and protein for design the new lead compounds for research. GlideScore is a practical scoring function that estimates the ligand binding free energy, including force field i.e. electrostatic, van der Waals etc, assistance and terms rewarding or penalizing interactions known to influence ligand binding. It has been enhanced for prediction of docking accuracy, database enrichment and binding affinity. ${ }^{20}$

\section{MATERIALS AND METHODS}

All chemicals used in synthesis and antimicrobial activity were acquired from Himedia Laboratories and TM Media, Titan Biotech Ltd., Delhi. Cefadroxil, Ofloxacin and Fluconazole were gathered from $\mathrm{CDH}$, Mumbai. All strains were seized from MTCC, Chandigarh. Chemical reactions were sustained under surveillance by TLC on silica gel plate; spotting was investigated in iodine and UV chambers, in mobile media Methanol: Ethyl Acetate: Hexane (0.5:3.5:6). For detection of melting points sonar melting point apparatus in open capillary tubes were used. ${ }^{1} \mathrm{H}$ NMR and ${ }^{13} \mathrm{C}$ NMR spectra were proved in DMSO and deuterated $\mathrm{CDCl}_{3}$ on Bruker Avance II 400 NMR spectrometer at a frequency of $400 \mathrm{MHz}$ and $100 \mathrm{MHz}$ downfield to tetramethyl silane standard. Coupling constants $(J)$ and chemical shifts were noted in Hertz $(\mathrm{Hz})$ and $\mathrm{d}$ (parts per million). Mass spectrums were recorded by Waters Micromass Q-ToF Micro instrument. MIC were concluded in BOD incubator (Haryana Scientific and Engg. Corporation Ltd., Rohtak) and Laminar Air Flow biosafety cabinet (Hicon, New Delhi) respectively.

Synthesisof 2-(3-methylbenzo[b]thiophen-6-yl)-1-(4-nitrophenyl)-1H-benzo[d]imidazole-5-carboxylic acid outlined in Scheme 1 (Figure 1). ${ }^{21,22}$ Structural part of compounds was in confirmed by Mass spectra, ${ }^{23}{ }^{1} \mathrm{H}$ NMR and ${ }^{13} \mathrm{CNMR}^{24}$ and elemental analysis which were found in full agreement with their structures. Detection of reaction completion was done by single spot TLC under UV lamp. ${ }^{25}$

For the synthesis:1 mole of PEG 5000, 1.2 mole of 4 - fluro 3 - nitro benzoic acid [2], 1.2 mole of DCC and a pinch of DMAP is dissolved in DCM and kept for stirring for two days at room temperature. To the precipitated product [3] add 1.1 mole of 4-nitrobenzenamine and then stirred for one day which is precipitated by ether. The product [4] was then reacted with 1.2 moles 4-MBA, 1.2 moles of DCC, pinch of DMAP dissolved in the solvent DCM then stirred for one day. Retrieved product [5] is subjected to reduction by adding the mixture of 1 mole of $\mathrm{NH}_{4} \mathrm{Cl}$ and 1 mole of $\mathrm{Zn}$, in methanol and stirred for $3 \mathrm{hrs}$, centrifuge the reaction mixture and filter to remove $\mathrm{Zn}$. Then 


\section{Table 1: Physical data of title compounds.}

\begin{tabular}{|c|c|c|c|c|c|c|}
\hline Sr. No. & Different Compounds & $\begin{array}{l}\text { Molecular } \\
\text { Formula }\end{array}$ & $\begin{array}{l}\text { Molecular } \\
\text { Weight }\end{array}$ & $\begin{array}{l}\text { Melting } \\
\text { Points }\left({ }^{\circ} \mathrm{C}\right)\end{array}$ & $\begin{array}{c}\% \\
\text { Yields }\end{array}$ & $R_{f}$ \\
\hline \multirow[t]{2}{*}{ C } & $\begin{array}{l}\text { 1-(2,4-dinitrophenyl)-2-(3-methylbenzo[b]thiophen- } \\
\text { 6-yl)-1H-benzo[d]imidazole-5-carboxylic acid }\end{array}$ & $\mathrm{C}_{23} \mathrm{H}_{15} \mathrm{~N}_{3} \mathrm{O}_{4} \mathrm{~S}$ & 429.45 & $120-122$ & 84.4 & 0.36 \\
\hline & Different Anilines (R) & & & & & \\
\hline C-1 & naphthalen-1-amine & $\mathrm{C}_{33} \mathrm{H}_{22} \mathrm{~N}_{4} \mathrm{O}_{3} \mathrm{~S}$ & 554.62 & 102-103 & 80.12 & 0.59 \\
\hline $\mathrm{C}-2$ & 2,3-difluoroaniline & $\mathrm{C}_{29} \mathrm{H}_{18} \mathrm{~F}_{2} \mathrm{~N}_{4} \mathrm{O}_{3} \mathrm{~S}$ & 540.54 & $132-134$ & 68.75 & 0.65 \\
\hline $\mathrm{C}-3$ & 2,4-dimethylaniline & $\mathrm{C}_{31} \mathrm{H}_{24} \mathrm{~N}_{4} \mathrm{O}_{3} \mathrm{~S}$ & 532.61 & $162-164$ & 82.34 & 0.77 \\
\hline C-4 & 2,4-dinitroaniline & $\mathrm{C}_{29} \mathrm{H}_{18} \mathrm{~N}_{6} \mathrm{O}_{7} \mathrm{~S}$ & 594.55 & $242-244$ & 78 & 0.52 \\
\hline C-5 & 2,5-dichloroaniline & $\mathrm{C}_{29} \mathrm{H}_{18} \mathrm{Cl}_{2} \mathrm{~N}_{4} \mathrm{O}_{3} \mathrm{~S}$ & 573.45 & $92-94$ & 85.65 & 0.44 \\
\hline C-6 & 2,5-difluoroaniline & $\mathrm{C}_{29} \mathrm{H}_{18} \mathrm{~F}_{2} \mathrm{~N}_{4} \mathrm{O}_{3} \mathrm{~S}$ & 540.54 & $99-101$ & 80.4 & 0.55 \\
\hline C-7 & 2,4,6-trimethylaniline & $\mathrm{C}_{32} \mathrm{H}_{26} \mathrm{~N}_{4} \mathrm{O}_{3} \mathrm{~S}$ & 546.64 & 163-165 & 85.92 & 0.8 \\
\hline C-8 & 2,6-difluoroaniline & $\mathrm{C}_{29} \mathrm{H}_{18} \mathrm{~F}_{2} \mathrm{~N}_{4} \mathrm{O}_{3} \mathrm{~S}$ & 540.54 & 187-189 & 75.75 & 0.6 \\
\hline C-9 & 2-bromo-4-methylaniline & $\mathrm{C}_{30} \mathrm{H}_{21} \mathrm{BrN}_{4} \mathrm{O}_{3} \mathrm{~S}$ & 597.48 & 173-179 & 81.9 & 0.7 \\
\hline$C-10$ & 2-bromo-5-nitroaniline & $\mathrm{C}_{29} \mathrm{H}_{18} \mathrm{BrN}_{5} \mathrm{O}_{5} \mathrm{~S}$ & 628.45 & $228-230$ & 92.5 & 0.81 \\
\hline C-11 & 2-chloro-4-methylaniline & $\mathrm{C}_{30} \mathrm{H}_{21} \mathrm{CIN}_{4} \mathrm{O}_{3} \mathrm{~S}$ & 553.03 & $168-170$ & 72.69 & 0.62 \\
\hline C-12 & 2-chloro-4-nitroaniline & $\mathrm{C}_{29} \mathrm{H}_{18} \mathrm{CIN}_{5} \mathrm{O}_{5} \mathrm{~S}$ & 584 & $167-169$ & 83.54 & 0.5 \\
\hline$C-13$ & 2-chloroaniline & $\mathrm{C}_{29} \mathrm{H}_{19} \mathrm{CIN}_{4} \mathrm{O}_{3} \mathrm{~S}$ & 539 & $152-154$ & 77.85 & 0.61 \\
\hline C-14 & 2-nitroaniline & $\mathrm{C}_{29} \mathrm{H}_{19} \mathrm{~N}_{5} \mathrm{O}_{5} \mathrm{~S}$ & 549.56 & $124-126$ & 70.16 & 0.71 \\
\hline C-15 & o-toluidine & $\mathrm{C}_{30} \mathrm{H}_{22} \mathrm{~N}_{4} \mathrm{O}_{3} \mathrm{~S}$ & 518.59 & $161-163$ & 82.5 & 0.89 \\
\hline C-16 & 3-nitroaniline & $\mathrm{C}_{29} \mathrm{H}_{19} \mathrm{~N}_{5} \mathrm{O}_{5} \mathrm{~S}$ & 549.56 & 183-185 & 75.6 & 0.79 \\
\hline C-17 & 3-(trifluoromethyl)aniline & $\mathrm{C}_{30} \mathrm{H}_{19} \mathrm{~F}_{3} \mathrm{~N}_{4} \mathrm{O}_{3} \mathrm{~S}$ & 572.56 & 179-181 & 72.65 & 0.42 \\
\hline $\mathrm{C}-18$ & 4-bromo-2-methylaniline & $\mathrm{C}_{30} \mathrm{H}_{21} \mathrm{BrN}_{4} \mathrm{O}_{3} \mathrm{~S}$ & 597.48 & 153-155 & 66.8 & 0.67 \\
\hline C-19 & 4-fluoro-3-methylaniline & $\mathrm{C}_{30} \mathrm{H}_{21} \mathrm{FN}_{4} \mathrm{O}_{3} \mathrm{~S}$ & 536.58 & $206-208$ & 85.7 & 0.76 \\
\hline C-20 & 4-nitroaniline & $\mathrm{C}_{29} \mathrm{H}_{19} \mathrm{~N}_{5} \mathrm{O}_{5} \mathrm{~S}$ & 549.56 & $276-278$ & 76.5 & 0.51 \\
\hline C-21 & 5-tert-butyl-2-methoxyaniline & $\mathrm{C}_{34} \mathrm{H}_{30} \mathrm{~N}_{4} \mathrm{O}_{4} \mathrm{~S}$ & 590.69 & $188-190$ & 74.8 & 0.63 \\
\hline C-22 & Phenylmethanamine & $\mathrm{C}_{30} \mathrm{H}_{22} \mathrm{~N}_{4} \mathrm{O}_{3} \mathrm{~S}$ & 518.59 & $159-161$ & 85.4 & 0.74 \\
\hline C-23 & 4-chloro-2-nitroaniline & $\mathrm{C}_{29} \mathrm{H}_{18} \mathrm{CIN}_{5} \mathrm{O}_{5} \mathrm{~S}$ & 585.07 & 209-211 & 80.9 & 0.69 \\
\hline
\end{tabular}

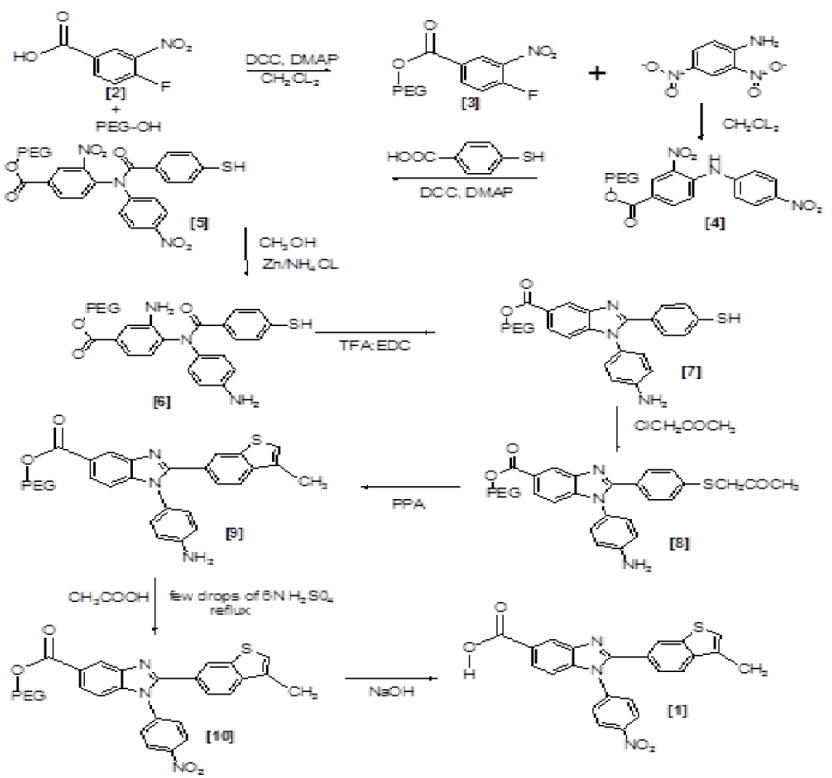

Figure 1: Scheme 1. Synthetic route for 1-(4-nitrophenyl)2-(3-methylbenzzo[b]thiophen-6-yl)-1h-benzo[d]imidazole5-carboxylic acid.
20 to $30 \mathrm{ml}$ of DCM was added and again centrifuged to remove $\mathrm{NH}_{4} \mathrm{Cl}$. Product obtained [6] was treated with TFA:EDC in 1:10 ratio and then stirred for one day. Product obtained [7] was treated with 1.3molesof chloroacetone and stirred for one day. Then product [8] was reacted with 1.4 moles of PPA and stirred for one day. Product recovered [9] was treated with 1.2 moles tetra-n-propylammoniumbromate in the $20 \mathrm{ml}$ of glacial acetic acid and $5 \mathrm{ml}$ of $6 \mathrm{~N}$ sulfuric acid and refluxed for $6 \mathrm{hrs}$ and product was filtered/washed with the help of water. Polymer was cleaved by treating the product recovered [10] with $0.5 \mathrm{~g}$ of sodium hydroxide by dissolving in methanol with stirring to get final product of 2-(3-methylbenzo[b]thiophen-6-yl)1-(4-nitrophenyl)-1H-benzo[d]imidazole-5-carboxylic acid.

Note: After each step product was recovered by precipitating with diethyl ether. 


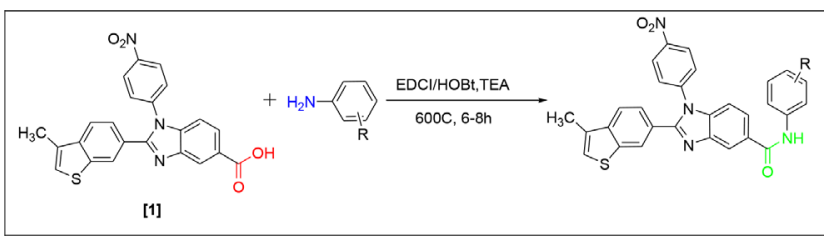

Figure 2: Scheme 2. Amide derivatives of 2-(3-methylbenzo[b] thiophen-6-yl)-1-(4-nitrophenyl)-1H-benzo[d]imidazole-5-carboxylic acid.

Total 100 amide derivatives were designed using Chemdraw and docking studies were carried out, out of which superlative 23 compounds were selected based on docking score.

Amide library of 2-(3-methylbenzo[b]thiophen6-yl)-1-(4-nitrophenyl)-1H-benzo[d] imidazole5-carboxylic acid outlined in Scheme 2 (Figure 2): ${ }^{26}$ 2-(3-methylbenzo[b]thiophen-6-yl)-1-(4-nitrophenyl)1H-benzo[d]imidazole-5-carboxylic acid $(0.27 \mathrm{mmol}$, 1.0 equiv) [1] was taken in the respective $\mathrm{RBF}$. To each $\mathrm{RBF}$, corresponding aromatic amines (C-1 to $\mathrm{C}-23$ ) (1.2 equiv) (Table 2 ) was added followed by DMF (2 $\mathrm{ml}$ ) and TEA (54 mg, $0.54 \mathrm{mmol}, 2.0$ equiv), HOBt (0.54mmol, 2equiv) and EDCI (0.54mmol, 2equiv). $\mathrm{RBF}$ were reserved in magnetic stirrer with hot plate for $6-8 \mathrm{~h}$ for heating at $60^{\circ} \mathrm{C}$ with stirring. After the reaction was completed (TLC monitoring), $5-7 \mathrm{ml}$ of an aqueous saturated solution of sodium hydrogen carbonate was added into each reaction RBF. The quenched reaction mixtures were either filtered by using filter paper, for liquid-liquid extraction, water $(8-10 \mathrm{ml})$ was added into each reaction mixture and extracted using organic solvent $(8-10 \mathrm{ml})$. The combined organic layer washed with water, separated/filtered using whatman filter paper, dried over anhydrous sodium sulphate and concentrated by using Rotary evaporator. Samples were purified by using column chromatography (using an optimized mixture of Ethyl acetate: Hexane as an eluent).

\section{Antimicrobial activity: Minimum inhibitory concentrations (MIC):}

The tube dilution method was used for the determination of microbial inhibitory potential of the synthesized derivatives against E. coli MTCC 45, T. thermophilus MTCC 1494, S. aureus MTCC 3160, B. subtilis MTCC 441, C. albicans MTCC 183 and A. niger MTCC 282 strains. Dilutions of standard and test compounds were processed in double strength nutrient broth I.P. (bacteria) or Sabouraud dextrose broth I.P. (fungi). ${ }^{27,28}$ The slants of B. subtilis MTCC 441, S. aureus MTCC 3160, E. coli MTCC 45 and T. thermophilus MTCC 1494, were incubated at the $30-35^{\circ} \mathrm{C}$ for $24 \mathrm{hr}$. The slants of $C$. albicans were incubated at $20-25^{\circ} \mathrm{C}$ for $48 \mathrm{hr}$ whereas; the slants of $A$. niger were incubated at $20-25^{\circ} \mathrm{C}$ for 5 days. After the incubation period sterilized $0.9 \% \mathrm{NaCl}$ solution was used to harvest the bacterial and fungal cultures from agar slant through suitable agitation and then the suspensions of micro-organisms were diluted with the sterile $0.9 \% \mathrm{NaCl}$ solution to cfu count was adjusted by adjusting the density of micro-organism suspension to that of $0.5 \mathrm{McF}$ arland standards by adding distilled water. The number of cfu was resolved by dilution pour-plate method. ${ }^{29}$ The samples were incubated at $37^{\circ} \mathrm{C}$ for $24 \mathrm{hr}$ (bacteria), at $25^{\circ} \mathrm{C}$ for 7 days $(A$. niger $)$ and at $37^{\circ} \mathrm{C}$ for $48 \mathrm{hr}$ (C. albicans) and the results were reported in terms of minimum inhibitory concentration (MIC).

\section{In silico Molecular docking studies: Docking achieved by Schrodinger Glide}

The Schrodinger, Inc. (New York, USA) software was exploited to study out the computational calculations and docking studies. All the computational work was accomplished in the Laboratory for Enzyme Inhibition Studies, Department of Pharmaceutical Sciences and Natural Products, Central University of Punjab, Bathinda, INDIA. Software ensures that protein and ligands amended form for docking, the receptor-grid files were engendered by grid-receptor generation program Glide. Grid-based Ligand Docking uses a stratified sequence of filters to fabricatepotential conformations of the ligand in the active site region of the protein receptor. At this stage, rudimentary score values and geometric filters were equipped out unlikely binding modes. ${ }^{30}$

Docking achieved by Autodock Vina 4.2: By using equipped PDB ID grid implemented molecular docking with .mol2-Tripos files by using Command prompt and after the compilation of docking score then confirm the interaction/hydrogen bond distance between molecules with PDB ID grid with the help of Autodock Vina 4.2.

Protein preparation: X-ray protein structure coordinates for aminoacyl-tRNA synthetase (AaRS) and tyrosyl-tRNA synthetase were downloaded from Protein Data Bank and were equipped with the help of the protein preparation wizard of Schrödinger (Prepwiz) (67). PDB ID: 1wny having resolution: $1.6 \mathrm{~A}^{0}$ and PDB ID: 1 jil having resolution: $2.2 \mathrm{~A}^{0}$ (selection criteria used was lowest resolution and availability) was exploited for the study. All the waters molecules (except those coordinated to metals) were separated except water present between the ligand and protein. The energycontrolled of the protein structure target site optimi- 


\begin{tabular}{|c|c|c|c|c|c|c|}
\hline \multirow[t]{3}{*}{ Compounds } & \multicolumn{6}{|c|}{ pMIC values in $\mu \mathrm{M} / \mathrm{ml}$} \\
\hline & \multicolumn{4}{|c|}{ Bacterial Strains } & \multicolumn{2}{|c|}{ Fungal Strains } \\
\hline & B. subtilis & S. aureus & E. coli & T. thermophilus & C. albicans & A. Niger \\
\hline C & 1.84 & 1.84 & 1.84 & 2.14 & 1.84 & 1.84 \\
\hline C-1 & 2.55 & 2.55 & 2.25 & 2.25 & 2.25 & 2.25 \\
\hline C-2 & 1.64 & 1.64 & 1.64 & 1.33 & 1.64 & 1.33 \\
\hline C-3 & 1.63 & 1.63 & 1.63 & 1.93 & 1.32 & 1.32 \\
\hline C-4 & 1.68 & 1.68 & 1.68 & 1.98 & 1.68 & 1.98 \\
\hline C-5 & 2.26 & 2.26 & 2.26 & 2.26 & 2.26 & 1.96 \\
\hline C-6 & 2.24 & 2.24 & 2.24 & 2.24 & 1.94 & 1.94 \\
\hline C-7 & 1.64 & 1.64 & 1.94 & 1.64 & 1.34 & 1.34 \\
\hline C-8 & 1.64 & 1.64 & 1.64 & 1.64 & 1.33 & 1.33 \\
\hline C-9 & 1.98 & 1.98 & 1.98 & 1.98 & 1.98 & 1.67 \\
\hline$C-10$ & 2.61 & 2.61 & 2.61 & 2.61 & 2.61 & 2.61 \\
\hline C-11 & 1.95 & 1.95 & 1.95 & 1.65 & 1.95 & 1.65 \\
\hline C-12 & 1.67 & 1.67 & 1.67 & 1.67 & 1.67 & 1.67 \\
\hline C-13 & 1.94 & 1.94 & 1.94 & 1.63 & 1.63 & 1.63 \\
\hline C-14 & 1.94 & 1.94 & 1.94 & 1.64 & 1.94 & 1.64 \\
\hline C-15 & 1.68 & 1.68 & 1.68 & 1.68 & 1.68 & 1.68 \\
\hline C-16 & 1.94 & 1.94 & 1.94 & 1.64 & 1.64 & 1.64 \\
\hline C-17 & 1.96 & 1.96 & 1.96 & 1.96 & 1.96 & 1.96 \\
\hline C-18 & 2.28 & 2.28 & 2.28 & 1.98 & 1.98 & 1.98 \\
\hline C-19 & 2.54 & 2.54 & 2.54 & 2.54 & 1.93 & 1.93 \\
\hline C-20 & 1.64 & 1.64 & 1.64 & 1.34 & 1.04 & 1.04 \\
\hline C-21 & 1.67 & 1.67 & 1.67 & 1.37 & 1.67 & 1.67 \\
\hline C-22 & 1.68 & 1.68 & 1.38 & 1.38 & 1.02 & 1.02 \\
\hline C-23 & 2.27 & 2.27 & 2.27 & 2.27 & 2.27 & 2.27 \\
\hline Cefadroxil & 1.72 & 3.44 & 3.44 & 3.44 & 0 & 0 \\
\hline Ofloxacin & 0.44 & 0.44 & 0.44 & 0.44 & 0 & 0 \\
\hline Fluconazole & 0 & 0 & 0 & 0 & 4.08 & 4.08 \\
\hline
\end{tabular}

zation of targated protein aminoacyl-tRNA synthetase (AaRS) and tyrosyl-tRNA synthetase were completed using OPLS-2005 as force field.

Ligand preparation: The three-dimensional structural library was generated using the Chemdraw software and then save as.mol2-Tripos. The 3-D structures of the selected ligands were anticipated for energy minimization using the LigPrep tool from Schrodinger to correct the coordinates, ionization, stereochemistry and tautomeric structure to gain the suitable conformation through the addition or deletion of hydrogen bonds. The ligands prepared after energy minimization were recycled for molecular docking studies.

\section{RESULTS AND DISCUSSION}

Physical and analytical data of synthesized derivatives are presented in Table 1.

Spectral Data: C: 2-(3-methylbenzo[b]thiophen-6-yl)1-(4-nitrophenyl)-1H-benzo[d]imidazole-5-carboxylic acid:MS: $430.45\left(\mathrm{M}^{+1}\right) .{ }^{1} \mathrm{H}$ NMR: $\delta 13.02$ (s, 1H), 8.50 $(\mathrm{s}, 1 \mathrm{H}), 8.28(\mathrm{~d}, J=7.8 \mathrm{~Hz}, 2 \mathrm{H}), 8.16(\mathrm{~s}, 1 \mathrm{H}), 8.04$ $(\mathrm{d}, J=10.2 \mathrm{~Hz}, 3 \mathrm{H}), 7.88(\mathrm{~d}, J=7.9 \mathrm{~Hz}, 2 \mathrm{H}), 7.83$ $(\mathrm{d}, J=8.1 \mathrm{~Hz}, 1 \mathrm{H}), 7.24$ (s, 1H), $2.41(\mathrm{~s}, 3 \mathrm{H}) \cdot{ }^{13} \mathrm{C}$ NMR: $\delta 167.55,155.26,144.34,143.96,142.17,139.67,139.53$, 138.26, 138.07, 131.30, 130.89, 128.40, 127.71, 127.29, 127.21, 126.67, 124.49, 124.32, 122.42, 122.08, 121.69, $108.82,14.17$. 
C-1: 2-(3-methylbenzo[b]thiophen-6-yl)-N-(naphthalen1-yl)-1-(4-nitrophenyl)-1H-benzo[d]imidazole-5-carboxamide: MS: $555.14\left(\mathrm{M}^{+1}\right) .{ }^{1} \mathrm{H}$ NMR: $\delta 10.39(\mathrm{~s}, 1 \mathrm{H})$, $8.46(\mathrm{~s}, 1 \mathrm{H}), 8.28$ (d, J = 7.9 Hz, 2H), $8.16(\mathrm{~s}, 1 \mathrm{H}), 8.04$ $(\mathrm{d}, J=8.4 \mathrm{~Hz}, 2 \mathrm{H}), 7.92(\mathrm{t}, J=10.8 \mathrm{~Hz}, 3 \mathrm{H}), 7.85$ $(\mathrm{t}, J=8.5 \mathrm{~Hz}, 3 \mathrm{H}), 7.68(\mathrm{~d}, J=7.4 \mathrm{~Hz}, 1 \mathrm{H}), 7.63$ $(\mathrm{d}, J=7.6 \mathrm{~Hz}, 1 \mathrm{H}), 7.54(\mathrm{t}, J=7.2 \mathrm{~Hz}, 2 \mathrm{H}), 7.28$ (t, $J=8.0 \mathrm{~Hz}, 1 \mathrm{H}), 7.24(\mathrm{~s}, 1 \mathrm{H}), 2.41(\mathrm{~s}, 3 \mathrm{H}) \cdot{ }^{13} \mathrm{C}$ NMR: $\delta 166.76,158.19,158.11,155.67,155.59,154.00,147.11$, 143.01, 139.67, 139.53, 137.23, 136.02, 130.89, 130.73, $130.48,130.02,128.10,127.94,127.29,124.50,124.32$, 123.86, 122.43, 121.87, 121.32, 119.86, 118.96, 111.51, 111.48, 111.31, 111.28, 109.11, 14.17.

C-2: N-(2,3-difluorophenyl)-2-(3-methylbenzo[b]thiophen-6-yl)-1-(4-nitrophenyl)-1H-benzo[d]imidazole5-carboxamide: MS: $541.11\left(\mathrm{M}^{+1}\right) .{ }^{1} \mathrm{H}$ NMR: $\delta 10.94$ $(\mathrm{s}, 1 \mathrm{H}), 8.43(\mathrm{~s}, 1 \mathrm{H}), 8.28(\mathrm{~d}, J=7.9 \mathrm{~Hz}, 2 \mathrm{H}), 8.23$ $(\mathrm{s}, 1 \mathrm{H}), 8.04(\mathrm{~d}, J=10.1 \mathrm{~Hz}, 1 \mathrm{H}), 7.98(\mathrm{~d}, J=9.1 \mathrm{~Hz}, 2 \mathrm{H})$, $7.88(\mathrm{t}, J=11.1 \mathrm{~Hz}, 3 \mathrm{H}), 7.73(\mathrm{~d}, J=8.0 \mathrm{~Hz}, 1 \mathrm{H}), 7.24$ $(\mathrm{s}, 1 \mathrm{H}), 7.15$ (q, $J=7.6 \mathrm{~Hz}, 1 \mathrm{H}), 6.97$ (q, $J=7.2 \mathrm{~Hz}$, $1 \mathrm{H}), 2.41$ (s, 3H). ${ }^{13} \mathrm{C}$ NMR: $\delta$ 164.82, 155.26, 144.34, 143.96, 141.98, 139.67, 139.53, 138.26, 136.33, 135.86, 131.84, 131.30, 130.89, 130.80, 130.45, 128.40, 127.71, 127.37, 127.29, 126.64, 125.18, 124.49, 124.32, 123.81, 122.42, 122.08, 119.78, 109.57, 14.17 .

C-3: N-(2,4-dimethylphenyl)-2-(3-methylbenzo[b] thiophen-6-yl)-1-(4-nitrophenyl)-1H-benzo[d]imidazole-5-carboxamide: MS: $533.16\left(\mathrm{M}^{+1}\right) \cdot{ }^{1} \mathrm{H}$ NMR: $\delta 9.35$ (s, 1H), 8.45 (s, 1H), 8.28 (d, $J=7.9 \mathrm{~Hz}, 2 \mathrm{H}), 8.23$ (s, 1H), 8.03 (d, J = 9.2 Hz, 1H), 7.98 (d, J = 7.5 Hz, 1H), $7.92(\mathrm{t}, J=11.7 \mathrm{~Hz}, 3 \mathrm{H}), 7.73(\mathrm{~d}, J=8.0 \mathrm{~Hz}, 1 \mathrm{H})$, $7.24(\mathrm{~s}, 1 \mathrm{H}), 7.04(\mathrm{~d}, J=6.8 \mathrm{~Hz}, 1 \mathrm{H}), 6.99(\mathrm{~d}, J=9.9 \mathrm{~Hz}$, 2H), 2.40 (s, 3H), $2.20(\mathrm{~d}, J=5.7 \mathrm{~Hz}, 6 \mathrm{H}) .{ }^{13} \mathrm{C}$ NMR: $\delta$ 164.84, 154.00, 149.94, 147.11, 144.42, 143.01, 139.67, $139.53,137.23,136.02,130.89,130.73,130.44,130.02$, 127.94, 127.29, 124.50, 124.32, 123.86, 122.43, 121.87, $121.32,119.86,119.73,115.38,112.31,109.11,56.01$, 34.53, 31.27, 14.17 .

C-4: N-(2,4-dinitrophenyl)-2-(3-methylbenzo[b]thiophen -6-yl)-1-(4-nitrophenyl)-1H-benzo[d]imidazole-5-carboxamide: MS: $595.10\left(\mathrm{M}^{+1}\right) .{ }^{1} \mathrm{H}$ NMR: $\delta 11.61$ (s, 1H), 8.78 (s, $1 \mathrm{H}), 8.58$ (d, $J=8.5 \mathrm{~Hz}, 1 \mathrm{H}), 8.47$ (s, 1H), $8.28(\mathrm{~d}, J=7.8 \mathrm{~Hz}, 2 \mathrm{H}), 8.24(\mathrm{~s}, 1 \mathrm{H}), 8.12-8.01$ $(\mathrm{m}, 2 \mathrm{H}), 7.99(\mathrm{~d}, J=6.0 \mathrm{~Hz}, 2 \mathrm{H}), 7.90(\mathrm{~d}, J=7.8 \mathrm{~Hz}, 2 \mathrm{H})$, 7.73 (d, $J=7.9 \mathrm{~Hz}, 1 \mathrm{H}), 7.24$ (s, 1H), 2.41 (s, 3H). ${ }^{13} \mathrm{C}$ NMR: $\delta 167.04,154.00,147.11,143.01,139.67,139.53$, 137.23, 136.02, 134.96, 133.66, 132.52, 130.89, 130.73, $130.48,130.02,129.15,127.94,127.29,124.50,124.32$, 123.86, 122.43, 121.87, 121.32, 119.86, 109.11, 20.74, $17.51,14.17$.

C-5: N-(2,5-dichlorophenyl)-2-(3-methylbenzo[b]thiophen-6-yl)-1-(4-nitrophenyl)-1H-benzo[d]imidazole- 5-carboxamide: MS: $574.04\left(\mathrm{M}^{+1}\right) .{ }^{1} \mathrm{H}$ NMR: $\delta 9.76(\mathrm{~s}$, $1 \mathrm{H}), 8.43(\mathrm{~s}, 1 \mathrm{H}), 8.28(\mathrm{~d}, J=7.8 \mathrm{~Hz}, 2 \mathrm{H}), 8.24(\mathrm{~s}, 1 \mathrm{H})$, $8.04(\mathrm{~d}, J=9.3 \mathrm{~Hz}, 1 \mathrm{H}), 7.98(\mathrm{~d}, J=7.9 \mathrm{~Hz}, 1 \mathrm{H}), 7.89$ $(\mathrm{d}, J=8.2 \mathrm{~Hz}, 3 \mathrm{H}), 7.84(\mathrm{~s}, 1 \mathrm{H}), 7.72(\mathrm{~d}, J=7.7 \mathrm{~Hz}, 1 \mathrm{H})$, $7.50(\mathrm{~d}, J=7.6 \mathrm{~Hz}, 1 \mathrm{H}), 7.24(\mathrm{~s}, 1 \mathrm{H}), 7.08(\mathrm{~d}, J=8.0 \mathrm{~Hz}$, 1H), 2.41 (s, 3H). ${ }^{13} \mathrm{C}$ NMR: $\delta$ 164.82, 154.00, 147.11, 143.01, 139.67, 139.53, 137.23, 136.02, 135.86, 131.84, $130.89,130.80,130.73,130.44,130.02,127.94,127.37$, $127.29,126.64,125.18,124.50,124.32,123.86,122.43$, 121.87, 121.32, 119.86, 109.11, 14.17 .

C-6: N-(2,5-difluorophenyl)-2-(3-methylbenzo[b]thiophen-6-yl)-1-(4-nitrophenyl)-1H-benzo[d]imidazole5-carboxamide: MS: $541.11\left(\mathrm{M}^{+1}\right) .{ }^{1} \mathrm{H}$ NMR: $\delta 10.72(\mathrm{~s}$, $1 \mathrm{H}), 8.44(\mathrm{~s}, 1 \mathrm{H}), 8.28(\mathrm{~d}, J=7.9 \mathrm{~Hz}, 2 \mathrm{H}), 8.24(\mathrm{~s}, 1 \mathrm{H})$, $8.04(\mathrm{~d}, J=9.2 \mathrm{~Hz}, 1 \mathrm{H}), 7.98(\mathrm{~d}, J=8.3 \mathrm{~Hz}, 2 \mathrm{H}), 7.89$ $(\mathrm{d}, J=8.6 \mathrm{~Hz}, 3 \mathrm{H}), 7.72(\mathrm{~d}, J=8.0 \mathrm{~Hz}, 1 \mathrm{H}), 7.34-7.26$ $(\mathrm{m}, 1 \mathrm{H}), 7.24(\mathrm{~s}, 1 \mathrm{H}), 6.95(\mathrm{t}, J=7.9 \mathrm{~Hz}, 1 \mathrm{H}), 2.41$ (s, $3 \mathrm{H}) .{ }^{13} \mathrm{C}$ NMR: $\delta 164.77,154.00,147.11,146.82,143.01$, 139.67, 139.53, 137.60, 137.23, 136.02, 135.00, 130.89, $130.73,130.44,130.02,127.94,127.29,124.50,124.32$, 123.86, 122.43, 121.87, 121.32, 119.86, 119.02, 118.16, 115.87, 109.11, 14.17.

C-7: N-mesityl-2-(3-methylbenzo[b]thiophen-6-yl)-1(4-nitrophenyl)-1H-benzo[d]imidazole-5-carboxamide: MS: $547.18\left(\mathrm{M}^{+1}\right) .{ }^{1} \mathrm{H}$ NMR: $\delta 9.48(\mathrm{~s}, 1 \mathrm{H}), 8.44(\mathrm{~s}, 1 \mathrm{H})$, $8.28(\mathrm{~d}, J=7.7 \mathrm{~Hz}, 2 \mathrm{H}), 8.23(\mathrm{~s}, 1 \mathrm{H}), 8.04(\mathrm{~d}, J=9.2 \mathrm{~Hz}$, $1 \mathrm{H}), 7.98(\mathrm{~d}, J=7.9 \mathrm{~Hz}, 1 \mathrm{H}), 7.90(\mathrm{~d}, J=8.4 \mathrm{~Hz}, 3 \mathrm{H})$, $7.73(\mathrm{~d}, J=8.0 \mathrm{~Hz}, 1 \mathrm{H}), 7.24(\mathrm{~s}, 1 \mathrm{H}), 6.88(\mathrm{~s}, 2 \mathrm{H}), 2.41$ (s, 3H), 2.27 (s, 3H), 2.08 (s, 6H). ${ }^{13} \mathrm{C}$ NMR: $\delta$ 164.74, 155.26, 144.34, 143.96, 141.98, 141.85, 139.67, 139.53, 138.26, 136.33, 131.30, 130.89, 130.45, 128.40, 128.05, $127.85,127.71,127.29,124.85,124.82,124.49,124.32$, 123.81, 122.42, 122.08, 119.78, 117.62, 117.54, 110.70, 110.62, 109.57, 14.17 .

C-8: N-(2,6-difluorophenyl)-2-(3-methylbenzo[b]thiophen-6-yl)-1-(4-nitrophenyl)-1H-benzo[d]imidazole5-carboxamide: MS: $541.11\left(\mathrm{M}^{+1}\right) .{ }^{1} \mathrm{H}$ NMR: $\delta 9.58$ $(\mathrm{s}, 1 \mathrm{H}), 8.45(\mathrm{~s}, 1 \mathrm{H}), 8.28(\mathrm{~d}, J=7.9 \mathrm{~Hz}, 2 \mathrm{H}), 8.24(\mathrm{~s}, 1 \mathrm{H})$, $8.04(\mathrm{~d}, J=9.3 \mathrm{~Hz}, 1 \mathrm{H}), 7.98(\mathrm{~d}, J=7.8 \mathrm{~Hz}, 1 \mathrm{H}), 7.89$ $(\mathrm{d}, J=8.4 \mathrm{~Hz}, 3 \mathrm{H}), 7.72(\mathrm{~d}, J=8.1 \mathrm{~Hz}, 1 \mathrm{H}), 7.22$ $(\mathrm{d}, J=13.9 \mathrm{~Hz}, 2 \mathrm{H}), 7.01(\mathrm{~d}, J=8.3 \mathrm{~Hz}, 2 \mathrm{H}), 2.41$ (s, 3H). ${ }^{13} \mathrm{C}$ NMR: $\delta 164.88,154.00,147.11,143.01,139.67$, 139.53, 137.23, 136.02, 135.67, 130.89, 130.73, 130.44, 130.02, 129.37, 128.01, 127.94, 127.29, 127.28, 125.85, $124.50,124.32,123.86,122.92,122.43,121.87,121.32$, 119.86, 109.11, 14.17.

C-9: N-(2-bromo-4-methylphenyl)-2-(3-methylbenzo[b] thiophen-6-yl)-1-(4-nitrophenyl)-1H-benzo[d]imidazole-5-carboxamide: MS: $598.05\left(\mathrm{M}^{+1}\right) .{ }^{1} \mathrm{H}$ NMR: $\delta 9.76$ (s, 1H), 8.44 (s, 1H), 8.28 (d, $J=7.8 \mathrm{~Hz}, 2 \mathrm{H}), 8.24$ (s, 1H), $8.04(\mathrm{~d}, J=9.3 \mathrm{~Hz}, 1 \mathrm{H}), 7.98(\mathrm{~d}, J=7.8 \mathrm{~Hz}, 1 \mathrm{H})$, $7.91(\mathrm{dd}, J=15.6,7.4 \mathrm{~Hz}, 4 \mathrm{H}), 7.72(\mathrm{~d}, J=7.9 \mathrm{~Hz}, 1 \mathrm{H})$, 
7.45 (s, 1H), 7.24 (s, 1H), 7.17 (d, $J=7.4 \mathrm{~Hz}, 1 \mathrm{H}), 2.40$ (s, 3H), 2.28 (s, $3 \mathrm{H}) .{ }^{13} \mathrm{C}$ NMR: $\delta 164.83,155.26,144.34$, 143.96, 141.98, 139.67, 139.53, 138.26, 136.33, 135.73, 135.40, 132.61, 131.30, 130.89, 130.45, 128.64, 128.40, $127.71,127.29,124.49,124.32,123.81,122.42,122.26$, 122.08, 119.78, 114.48, 109.57, 20.65, 14.17.

C-10: N-(2-bromo-5-nitrophenyl)-2-(3-methylbenzo[b] thiophen-6-yl)-1-(4-nitrophenyl)-1H-benzo[d]imidazole-5-carboxamide: MS: $629.02\left({ }^{\mathrm{M}+1}\right) .{ }^{1} \mathrm{H}$ NMR: $\delta 10.67$ $(\mathrm{s}, 1 \mathrm{H}), 8.92(\mathrm{~s}, 1 \mathrm{H}), 8.44(\mathrm{~s}, 1 \mathrm{H}), 8.28(\mathrm{~d}, J=7.9 \mathrm{~Hz}$, $2 \mathrm{H}), 8.16(\mathrm{~s}, 1 \mathrm{H}), 8.04(\mathrm{~d}, J=8.2 \mathrm{~Hz}, 2 \mathrm{H}), 7.98-7.89$ $(\mathrm{m}, 2 \mathrm{H}), 7.88(\mathrm{~d}, J=6.5 \mathrm{~Hz}, 2 \mathrm{H}), 7.84(\mathrm{~d}, J=9.7 \mathrm{~Hz}$, 2H), 7.24 (s, $1 \mathrm{H}), 2.41$ (s, 3H). ${ }^{13} \mathrm{C}$ NMR: $\delta$ 164.77, 155.26, 146.82, 144.34, 143.96, 141.98, 139.67, 139.53, 138.26, 137.60, 136.33, 135.00, 131.30, 130.89, 130.45, $128.40,127.71,127.29,124.49,124.32,123.81,122.42$, $122.08,119.78,119.02,118.16,115.87,109.57,14.17$.

C-11: N-(2-chloro-4-methylphenyl)-2-(3-methylbenzo [b]thiophen-6-yl)-1-(4-nitrophenyl)-1H-benzo[d]imidazole-5-carboxamide: MS: $554.11\left(\mathrm{M}^{+1}\right) .{ }^{1} \mathrm{H}$ NMR: $\delta$ 10.01 (s, 1H), 8.43 (s, 1H), 8.28 (d, $J=7.9 \mathrm{~Hz}, 2 \mathrm{H})$, $8.24(\mathrm{~s}, 1 \mathrm{H}), 8.04(\mathrm{~d}, J=9.1 \mathrm{~Hz}, 1 \mathrm{H}), 7.98(\mathrm{~d}, J=7.9 \mathrm{~Hz}$, 1H), $7.97-7.88(\mathrm{~m}, 2 \mathrm{H}), 7.87(\mathrm{~d}, J=9.9 \mathrm{~Hz}, 2 \mathrm{H}), 7.72$ $(\mathrm{d}, J=7.9 \mathrm{~Hz}, 1 \mathrm{H}), 7.46(\mathrm{~s}, 1 \mathrm{H}), 7.24(\mathrm{~s}, 1 \mathrm{H}), 7.08$ (d, $J=7.8 \mathrm{~Hz}, 1 \mathrm{H}), 2.40(\mathrm{~s}, 3 \mathrm{H}), 2.28(\mathrm{~s}, 3 \mathrm{H}) \cdot{ }^{13} \mathrm{C}$ NMR: $\delta$ 164.88, 155.26, 144.34, 143.96, 141.98, 139.67, 139.53, 138.26, 136.41, 136.33, 134.07, 131.30, 130.89, 130.45, $129.78,128.40,128.15,127.71,127.29,124.49,124.32$, 124.08, 123.81, 122.98, 122.42, 122.08, 119.78, 109.57, 20.35, 14.17 .

C-12: N-(2-chloro-4-nitrophenyl)-2-(3-methylbenzo[b] thiophen-6-yl)-1-(4-nitrophenyl)-1H-benzo[d]imidazole-5-carboxamide: MS: $585.07\left(\mathrm{M}^{+1}\right) .{ }^{1} \mathrm{H}$ NMR: $\delta 10.67$ $(\mathrm{s}, 1 \mathrm{H}), 8.43(\mathrm{~s}, 1 \mathrm{H}), 8.34(\mathrm{~s}, 1 \mathrm{H}), 8.26(\mathrm{dd}, J=16.8,7.4$ $\mathrm{Hz}, 4 \mathrm{H}), 8.13$ (d, $J=8.6 \mathrm{~Hz}, 1 \mathrm{H}), 8.04$ (d, $J=9.2 \mathrm{~Hz}$, 1H), $7.98(\mathrm{~d}, J=7.8 \mathrm{~Hz}, 1 \mathrm{H}), 7.88(\mathrm{t}, J=9.2 \mathrm{~Hz}, 3 \mathrm{H})$, $7.72(\mathrm{~d}, J=8.0 \mathrm{~Hz}, 1 \mathrm{H}), 7.24(\mathrm{~s}, 1 \mathrm{H}), 2.41(\mathrm{~s}, 3 \mathrm{H}) \cdot{ }^{13} \mathrm{C}$ NMR: $\delta$ 164.88, 155.26, 144.34, 143.96, 141.98, 140.00, $139.85,139.67,139.53,138.26,136.33,131.30,130.89$, $130.45,128.40,127.71,127.29,125.90,124.63,124.49$, $124.32,123.94,123.81,122.42,122.08,121.64,119.78$, 109.57, 14.17.

C-13: N-(2-chlorophenyl)-2-(3-methylbenzo[b]thiophen-6-yl)-1-(4-nitrophenyl)-1H-benzo[d]imidazole5-carboxamide: MS: $540.08\left(\mathrm{M}^{+1}\right) .{ }^{1} \mathrm{H}$ NMR: $\delta 10.13$ (s, $1 \mathrm{H}), 8.43$ (s, 1H), 8.28 (d, J = 7.9 Hz, 2H), $8.15(\mathrm{~s}, 1 \mathrm{H})$, $8.04(\mathrm{~d}, J=8.8 \mathrm{~Hz}, 2 \mathrm{H}), 7.91(\mathrm{~s}, 1 \mathrm{H}), 7.87(\mathrm{~d}, J=13.4$ $\mathrm{Hz}, 3 \mathrm{H}), 7.78(\mathrm{~d}, J=8.1 \mathrm{~Hz}, 1 \mathrm{H}), 7.54(\mathrm{~d}, J=7.9 \mathrm{~Hz}$, 1H), 7.38 (t, $J=7.8 \mathrm{~Hz}, 1 \mathrm{H}), 7.24(\mathrm{~s}, 1 \mathrm{H}), 6.97$ (t, $J=$ $7.8 \mathrm{~Hz}, 1 \mathrm{H}), 2.41$ (s, $3 \mathrm{H}) .{ }^{13} \mathrm{C}$ NMR: $\delta 164.88,155.26$, 144.34, 143.96, 141.98, 139.67, 139.53, 138.26, 136.33, 135.67, 131.30, 130.89, 130.45, 129.37, 128.40, 128.01,
127.71, 127.29, 127.28, 125.85, 124.49, 124.32, 123.81, 122.92, 122.42, 122.08, 119.78, 109.57, 14.17 .

C-14: 2-(3-methylbenzo[b]thiophen-6-yl)-N-(2-nitrophenyl) -1-(4-nitrophenyl)-1H-benzo[d]imidazole-5-carboxamide: MS: $550.11\left(\mathrm{M}^{+1}\right) .{ }^{1} \mathrm{H}$ NMR: $\delta 11.19(\mathrm{~s}, 1 \mathrm{H}), 8.44$ $(\mathrm{d}, J=7.8 \mathrm{~Hz}, 2 \mathrm{H}), 8.28(\mathrm{~d}, J=7.9 \mathrm{~Hz}, 2 \mathrm{H}), 8.15(\mathrm{~s}, 1 \mathrm{H})$, $8.04(\mathrm{t}, J=8.4 \mathrm{~Hz}, 2 \mathrm{H}), 7.90(\mathrm{~d}, J=8.6 \mathrm{~Hz}, 4 \mathrm{H}), 7.84$ $(\mathrm{d}, J=7.6 \mathrm{~Hz}, 1 \mathrm{H}), 7.69(\mathrm{t}, J=7.8 \mathrm{~Hz}, 1 \mathrm{H}), 7.22(\mathrm{~d}, J=$ $11.2 \mathrm{~Hz}, 2 \mathrm{H}), 2.41$ (s, 3H). ${ }^{13} \mathrm{C}$ NMR: $\delta 164.42,155.26$, 144.34, 143.96, 141.98, 139.67, 139.53, 138.26, 136.33, 135.80, 134.88, 131.30, 130.89, 130.45, 128.40, 127.87, 127.71, 127.29, 125.76, 124.49, 124.32, 123.81, 122.80, 122.42, 122.08, 119.78, 118.88, 109.57, 14.17 .

C-15: 2-(3-methylbenzo[b]thiophen-6-yl)-1-(4-nitrophenyl) -N-o-tolyl-1H-benzo[d]imidazole-5-carboxamide: MS: $519.14(\mathrm{M}+1) .{ }^{1} \mathrm{H}$ NMR: $\delta 9.45(\mathrm{~s}, 1 \mathrm{H}), 8.40(\mathrm{~s}, 1 \mathrm{H})$, $8.28(\mathrm{~d}, J=7.9 \mathrm{~Hz}, 2 \mathrm{H}), 8.23(\mathrm{~s}, 1 \mathrm{H}), 8.03(\mathrm{~d}, J=9.0 \mathrm{~Hz}$, $1 \mathrm{H}), 7.98(\mathrm{~d}, J=7.9 \mathrm{~Hz}, 1 \mathrm{H}), 7.91(\mathrm{t}, J=9.7 \mathrm{~Hz}, 3 \mathrm{H})$, $7.73(\mathrm{~d}, J=7.9 \mathrm{~Hz}, 1 \mathrm{H}), 7.45(\mathrm{~d}, J=8.4 \mathrm{~Hz}, 1 \mathrm{H}), 7.36(\mathrm{t}$, $J=7.7 \mathrm{~Hz}, 1 \mathrm{H}), 7.22(\mathrm{~d}, J=12.0 \mathrm{~Hz}, 3 \mathrm{H}), 2.40(\mathrm{~s}, 3 \mathrm{H})$, 2.20 (s, 3H). ${ }^{13} \mathrm{C}$ NMR: $\delta 165.00,155.26,144.34,143.96$, 141.98, 139.67, 139.53, 138.26, 136.33, 135.34, 132.70, $131.30,130.89,130.45,129.02,128.40,127.71,127.29$, $127.16,124.49,124.45,124.32,123.81,122.42,122.08$, 120.55, 119.78, 109.57, 17.80, 14.17 .

C-16: 2-(3-methylbenzo[b]thiophen-6-yl)-N-(3-nitrophenyl) -1-(4-nitrophenyl)-1H-benzo[d]imidazole-5-carboxamide: MS: $550.11\left(\mathrm{M}^{+1}\right) .{ }^{1} \mathrm{H}$ NMR: $\delta 10.14(\mathrm{~s}, 1 \mathrm{H}), 8.72$ $(\mathrm{s}, 1 \mathrm{H}), 8.41(\mathrm{~s}, 1 \mathrm{H}), 8.28(\mathrm{~d}, J=7.9 \mathrm{~Hz}, 2 \mathrm{H}), 8.17-8.09$ $(\mathrm{m}, 2 \mathrm{H}), 8.04(\mathrm{~d}, J=8.8 \mathrm{~Hz}, 3 \mathrm{H}), 7.91(\mathrm{t}, J=10.2 \mathrm{~Hz}$, $3 \mathrm{H}), 7.85(\mathrm{~d}, J=7.7 \mathrm{~Hz}, 1 \mathrm{H}), 7.62(\mathrm{t}, J=8.3 \mathrm{~Hz}, 1 \mathrm{H})$, 7.24 (s, $1 \mathrm{H}), 2.41$ (s, 3H). ${ }^{13} \mathrm{C}$ NMR: $\delta$ 165.00, 155.26, 144.34, 143.96, 141.98, 139.67, 139.53, 138.26, 136.33, 135.34, 132.70, 131.30, 130.89, 130.45, 129.02, 128.40, $127.71,127.29,127.16,124.49,124.45,124.32,123.81$, 122.42, 122.08, 120.55, 119.78, 109.57, 17.80, 14.17.

C-17: 2-(3-methylbenzo[b]thiophen-6-yl)-1-(4nitrophenyl)-N-(3-(trifluoromethyl) phenyl)-1Hbenzo[d]imidazole-5-carboxamide: MS: $550.11(\mathrm{M}+1)$. ${ }^{1} \mathrm{H}$ NMR: $\delta 9.39$ (s, 1H), 8.41 (s, 1H), 8.28 (d, $J=7.9$ $\mathrm{Hz}, 2 \mathrm{H}), 8.18$ (s, 1H), $8.07-7.98$ (m, 3H), 7.88 (dd, $J$ $=16.6,8.5 \mathrm{~Hz}, 4 \mathrm{H}), 7.79(\mathrm{~d}, J=7.9 \mathrm{~Hz}, 1 \mathrm{H}), 7.48(\mathrm{t}$, $J=7.8 \mathrm{~Hz}, 1 \mathrm{H}), 7.37(\mathrm{~d}, J=8.1 \mathrm{~Hz}, 1 \mathrm{H}), 7.24(\mathrm{~s}, 1 \mathrm{H})$, $2.41(\mathrm{~s}, 3 \mathrm{H}) .{ }^{13} \mathrm{C}$ NMR: $\delta$ 164.83, 154.00, 147.11, 143.01, 139.67, 139.53, 137.23, 136.02, 135.73, 135.40, 132.61, $130.89,130.73,130.44,130.02,128.64,127.94,127.29$, 124.50, 124.32, 123.86, 122.43, 122.26, 121.87, 121.32, 119.86, 114.48, 109.11, 20.65, 14.17.

C-18: N-(4-bromo-2-methylphenyl)-2-(3-methylbenzo [b] thiophen-6-yl)-1-(4-nitrophenyl)-1H-benzo[d]imidazole-5-carboxamide: MS: $598.05\left(\mathrm{M}^{+1}\right) .{ }^{1} \mathrm{H}$ NMR: $\delta$ $9.58(\mathrm{~s}, 1 \mathrm{H}), 8.39$ (s, 1H), 8.28 (d, $J=7.8 \mathrm{~Hz}, 2 \mathrm{H}), 8.23$ 
$(\mathrm{s}, 1 \mathrm{H}), 8.03(\mathrm{~d}, J=9.3 \mathrm{~Hz}, 1 \mathrm{H}), 7.98(\mathrm{~d}, J=7.8 \mathrm{~Hz}, 1 \mathrm{H})$, $7.90(\mathrm{~d}, J=7.7 \mathrm{~Hz}, 3 \mathrm{H}), 7.73(\mathrm{~d}, J=7.7 \mathrm{~Hz}, 1 \mathrm{H}), 7.64$ $(\mathrm{d}, J=7.1 \mathrm{~Hz}, 1 \mathrm{H}), 7.45(\mathrm{~s}, 1 \mathrm{H}), 7.29(\mathrm{~d}, J=6.9 \mathrm{~Hz}$, 1H), 7.24 (s, $1 \mathrm{H}), 2.40$ (s, 3H), 2.29 (s, 3H). ${ }^{13} \mathrm{C}$ NMR: $\delta$ 165.00, 155.26, 144.34, 143.96, 141.98, 139.67, 139.53, 138.26, 136.33, 135.12, 132.71, 131.30, 130.89, 130.84, $130.45,129.90,128.40,127.71,127.29,124.49,124.32$, $123.81,123.49,122.42,122.08,119.78,116.93,109.57$, 17.98, 14.17.

C-19: N-(4-fluoro-3-methylphenyl)-2-(3-methylbenzo [b]thiophen-6-yl)-1-(4-nitrophenyl)-1H-benzo[d]imidazole-5-carboxamide: MS: $537.14(\mathrm{M}+1) .{ }^{1} \mathrm{H}$ NMR: $\delta$ $9.46(\mathrm{~s}, 1 \mathrm{H}), 8.41(\mathrm{~s}, 1 \mathrm{H}), 8.28(\mathrm{~d}, J=7.8 \mathrm{~Hz}, 2 \mathrm{H}), 8.23$ (s, 1H), 8.04 (d, $J=9.3 \mathrm{~Hz}, 1 \mathrm{H}), 7.98$ (d, $J=7.9 \mathrm{~Hz}$, $1 \mathrm{H}), 7.88(\mathrm{dd}, J=14.8,8.6 \mathrm{~Hz}, 3 \mathrm{H}), 7.72(\mathrm{~d}, J=8.6$ $\mathrm{Hz}, 2 \mathrm{H}), 7.24(\mathrm{~s}, 1 \mathrm{H}), 7.19(\mathrm{~d}, J=6.4 \mathrm{~Hz}, 1 \mathrm{H}), 7.13(\mathrm{t}$, $J=7.8 \mathrm{~Hz}, 1 \mathrm{H}), 2.41(\mathrm{~s}, 3 \mathrm{H}), 2.25(\mathrm{~s}, 3 \mathrm{H}) \cdot{ }^{13} \mathrm{C}$ NMR: $\delta$ 164.83, 154.00, 147.11, 143.01, 139.67, 139.53, 137.23, $136.02,135.73,135.40,132.61,130.89,130.73,130.44$ 130.02, 128.64, 127.94, 127.29, 124.50, 124.32, 123.86, 122.43, 122.26, 121.87, 121.32, 119.86, 114.48, 109.11, 20.65, 14.17 .

C-20: $\quad$ 2-(3-methylbenzo[b]thiophen-6-yl)-N,1-bis(4nitrophenyl)-1H-benzo[d]imidazole-5-carboxamide: MS: $550.11\left(\mathrm{M}^{+1}\right) .{ }^{1} \mathrm{H}$ NMR: $\delta 8.41(\mathrm{~s}, 1 \mathrm{H}), 8.36(\mathrm{~s}, 1 \mathrm{H}), 8.29$ $(\mathrm{t}, J=6.5 \mathrm{~Hz}, 4 \mathrm{H}), 8.15(\mathrm{~s}, 1 \mathrm{H}), 8.02(\mathrm{~d}, J=8.7 \mathrm{~Hz}, 4 \mathrm{H})$, $7.93-7.84(\mathrm{~m}, 3 \mathrm{H}), 7.84$ (s, 1H), 7.24 (s, 1H), 2.41 (s, 3H). ${ }^{13} \mathrm{C}$ NMR: $\delta$ 165.61, 154.00, 148.71, 147.11, 143.01, 139.67, 139.53, 138.56, 137.23, 136.02, 131.13, 130.89, $130.73,130.51,130.02,127.94,127.29,126.44,124.50$, $124.32,123.86,122.43,121.87,121.32,119.86,118.21$, 114.96, 109.11, 14.17.

C-21: N-(5-tert-butyl-2-methoxyphenyl)-2-(3-methylbenzo [b]thiophen-6-yl)-1-(4-nitrophenyl)-1H-benzo[d]imidazole-5-carboxamide: MS: $591.20\left(\mathrm{M}^{+1}\right) .{ }^{1} \mathrm{H}$ NMR: $\delta$ $9.94(\mathrm{~s}, 1 \mathrm{H}), 8.42(\mathrm{~s}, 1 \mathrm{H}), 8.28(\mathrm{~d}, J=7.7 \mathrm{~Hz}, 2 \mathrm{H}), 8.23$ (s, $1 \mathrm{H}), 7.96$ (ddd, $J=35.4,17.6,8.3 \mathrm{~Hz}, 6 \mathrm{H}), 7.73$ (d, $J$ $=8.0 \mathrm{~Hz}, 1 \mathrm{H}), 7.24(\mathrm{~s}, 1 \mathrm{H}), 7.14(\mathrm{~d}, J=7.0 \mathrm{~Hz}, 1 \mathrm{H}), 6.91$ (d, $J=7.1 \mathrm{~Hz}, 1 \mathrm{H}), 3.83$ (s, 3H), 2.41 (s, 3H), 1.31 (s, 9H). ${ }^{13} \mathrm{C}$ NMR: $\delta 164.86,164.74,153.79,151.26,151.06$, 144.47, 144.37, 142.99, 142.22, 141.85, 139.67, 139.53, $135.81,130.89,130.72,130.44,128.05,127.85,127.29$, $125.19,124.85,124.82,124.79,124.50,124.32,123.86$, 122.43, 119.86, 117.62, 117.54, 110.70, 110.62, 109.13, 14.17 .

C-22: N-benzyl-2-(3-methylbenzo[b]thiophen-6-yl)1-(4-nitrophenyl)-1H-benzo[d]imidazole-5-carboxamide: MS: $519.14(\mathrm{M}+1) .{ }^{1} \mathrm{H}$ NMR: $\delta 8.46(\mathrm{~s}, 1 \mathrm{H}), 8.32$ $-8.21(\mathrm{~m}, 3 \mathrm{H}), 8.14(\mathrm{t}, J=9.1 \mathrm{~Hz}, 1 \mathrm{H}), 8.00(\mathrm{dd}, J=$ 17.3, 8.6 Hz, 2H), $7.91-7.81(\mathrm{~m}, 3 \mathrm{H}), 7.72(\mathrm{~d}, J=7.9$ $\mathrm{Hz}, 1 \mathrm{H}), 7.32$ (s, 4H), 7.24 (s, 1H), 4.65 (d, J = 8.9 Hz, 2H), 2.41 (s, 3H). ${ }^{13} \mathrm{C}$ NMR: $\delta$ 164.88, 154.00, 147.11,
143.01, 139.67, 139.53, 137.23, 136.41, 136.02, 134.07, $130.89,130.73,130.44,130.02,129.78,128.15,127.94$, $127.29,124.50,124.32,124.08,123.86,122.98,122.43$, 121.87, 121.32, 119.86, 109.11, 20.35, 14.17.

C-23: N-(4-chloro-2-nitrophenyl)-2-(3-methylbenzo[b] thiophen-6-yl)-1-(4-nitrophenyl)-1H-benzo[d]imidazole-5-carboxamide: MS: $519.14\left(\mathrm{M}^{+1}\right) .{ }^{1} \mathrm{H}$ NMR: $\delta 11.25$ (s, 1H), 8.43 (s, 1H), 8.28 (d, $J=7.8 \mathrm{~Hz}, 2 \mathrm{H}), 8.22$ $(\mathrm{s}, 1 \mathrm{H}), 8.05(\mathrm{~d}, J=11.9 \mathrm{~Hz}, 2 \mathrm{H}), 7.98(\mathrm{~d}, J=7.8 \mathrm{~Hz}, 1 \mathrm{H})$, $7.90(\mathrm{~d}, J=8.4 \mathrm{~Hz}, 3 \mathrm{H}), 7.73(\mathrm{~d}, J=7.6 \mathrm{~Hz}, 1 \mathrm{H}), 7.63$ $(\mathrm{d}, J=7.4 \mathrm{~Hz}, 1 \mathrm{H}), 7.56(\mathrm{~d}, J=7.6 \mathrm{~Hz}, 1 \mathrm{H}), 7.24$ (s, 1H), 2.41 (s, 3H). ${ }^{13} \mathrm{C}$ NMR: $\delta 164.42,154.00,147.11$, 143.01, 139.67, 139.53, 137.23, 136.02, 135.80, 134.88, $130.89,130.73,130.44,130.02,127.94,127.87,127.29$, $125.76,124.50,124.32,123.86,122.80,122.43,121.87$, 121.32, 119.86, 118.88, 109.11, 14.17.

\section{Antimicrobial activity}

\section{Minimum inhibitory concentrations (MIC)}

The synthesized derivatives were evaluated for their in vitro antibacterial activity against $S$. aureus, B. subtilis, E. coli, T. thermophilus and antifungal activity against $C$. albicans and $A$. niger. Antimicrobial activity of the synthesized compounds was concluded by the tube dilution method. From the reported pMIC values, it was noticed that compound $\mathrm{C}-1, \mathrm{C}-10$ and $\mathrm{C}-19$ was found to be most active against all the strains having pMIC C-1: 2.55, 2.55, 2.25, 2.25, 2.25, 2.25 and C-10: 2.61, 2.61, 2.61, 2.61, 2.61, 2.61 and C-19: 2.54, 2.54, 2.54, 2.54, $1.93,1.93 \mu \mathrm{M} / \mathrm{ml}$ value. Compound C-3 was found to be least antibacterial active and Compound C-22 was found tobe least antifungal active in the series. In general, the results of MIC studies exposed that the synthesized derivatives have bacteriocidal and fungicidal potential as comparted to standards Cefadroxil, Ofloxacin and Fluconazole. Here, Figure 3 graphically represent the pMIC, Table 2 represent the pMIC values of compounds with comparision to standards.

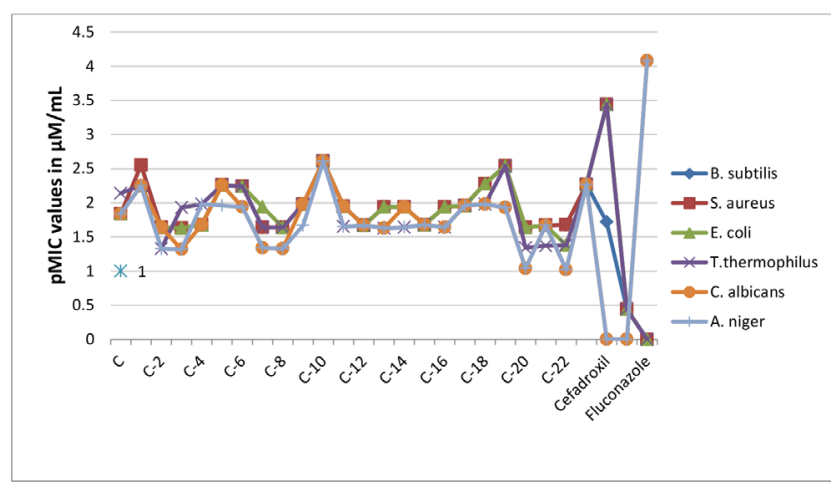

Figure 3: Antimicrobial activity (pMIC in $\mu \mathrm{M} / \mathrm{mL}$ ) of synthesized derivatives. 
Table 3: Docking Score of Synthesized Derivatives.

\begin{tabular}{|c|c|c|c|c|c|c|}
\hline \multirow[t]{3}{*}{ Sr No. } & \multirow{3}{*}{$\begin{array}{c}\begin{array}{c}\text { Docking Score by } \\
\text { Autodock Vina } 4.2 .\end{array} \\
\text { 1wny }\end{array}$} & & \multicolumn{4}{|c|}{ Docking Score by Schrodinger Glide } \\
\hline & & \multicolumn{2}{|c|}{ 1wny } & \multicolumn{2}{|c|}{ 1jil } & \multirow[b]{2}{*}{ glide gscore } \\
\hline & & $1 \mathrm{jil}$ & Docking Score & glide gscore & Docking Score & \\
\hline C-1 & -9.0 & -9.9 & -5.144 & -5.144 & -5.328 & -5.328 \\
\hline C-2 & -9.0 & -9.5 & -4.867 & -4.867 & -4.981 & -4.981 \\
\hline C-3 & -8.8 & -9.4 & -4.2 & -4.2 & -4.658 & -4.658 \\
\hline C-4 & -8.5 & -8.7 & -4.526 & -4.526 & -4.861 & -4.861 \\
\hline C-5 & -8.8 & -8.8 & -4.299 & -4.299 & -4.637 & -4.637 \\
\hline C-6 & -9.0 & -9.6 & -4.683 & -4.683 & -4.997 & -4.997 \\
\hline C-7 & -9.0 & -9.5 & -4.354 & -4.354 & -4.698 & -4.698 \\
\hline C-8 & -8.5 & -9.6 & -4.762 & -4.762 & -4.891 & -4.891 \\
\hline C-9 & -8.8 & -9.4 & -4.596 & -4.596 & -4.965 & -4.965 \\
\hline C-10 & -9.2 & -9.4 & -5.007 & -5.007 & -5.369 & -5.369 \\
\hline C-11 & -8.7 & -9.4 & -4.563 & -4.563 & -4.968 & -4.968 \\
\hline C-12 & -8.8 & -9.4 & -4.231 & -4.231 & -4.885 & -4.885 \\
\hline C-13 & -8.5 & -9.3 & -4.532 & -4.532 & -4.986 & -4.986 \\
\hline C-14 & -9.2 & -9.2 & -4.883 & -4.883 & -5.007 & -5.007 \\
\hline C-15 & -8.6 & -8.7 & -4.632 & -4.632 & -4.862 & -4.862 \\
\hline C-16 & -8.6 & -8.9 & -4.611 & -4.611 & -4.882 & -4.882 \\
\hline C-17 & -9.1 & -9.6 & -4.74 & -4.74 & -4.967 & -4.967 \\
\hline C-18 & -9.1 & -9.4 & -4.499 & -4.499 & -4.986 & -4.986 \\
\hline C-19 & -9.0 & -9.6 & -5.184 & -5.184 & -5.264 & -5.264 \\
\hline C-20 & -9.1 & -9.2 & -4.477 & -4.477 & -4.821 & -4.821 \\
\hline C-21 & -8.7 & -8.7 & -4.583 & -4.583 & -4.565 & -4.565 \\
\hline C-22 & -9.0 & -9.3 & -4.093 & -4.093 & -4.284 & -4.284 \\
\hline C-23 & -9.3 & -9.1 & -4.268 & -4.268 & -4.199 & -4.199 \\
\hline Cefadroxil & -10.2 & -10.6 & -5.847 & -5.847 & -5.895 & -5.895 \\
\hline Ofloxacin & -9.8 & -9.9 & -6.325 & -6.325 & -6.131 & -6.131 \\
\hline
\end{tabular}

Molecular Docking research: Newly designed and synthesized molecules/ligands along with standards were studied for their molecular docking behavior with the help of Schrodinger's Maestro package docking software and AutoDock Vina 4.2, Table 3. Ligands were docked was into binding modes and binding affinities to the 1wny.PDBQT and 1jil.PDBQT. Induced Fit Docking (IFD) method was utilized for the same. The predicted binding pattern exposed that synthesized ligands bind within catalytic cavity firmly via Hydrogen bond formation, pi-pi stacking and hydrophobic interaction. Molecular docking parameters and interaction pattern of ligands within the pocket.In the binding model the most active compounds are C-1, C-10 and C-19.

In compound C-1, $1 \mathrm{WNY}$ PDB,Visual inspection of the pose of structure umbrageous into the inhibitor binding cavity revealed that ligand is inserted in to "aromatic case” framed by PHE 324, TRP 232, THR 230, THR
233, VAL 318, GLY 316, THR 315, TRP 227, ASP 313 and the FAD aromatic ring of the protein (Figure 4) and 1JIL PDB, Visual inspection of the pose of structure umbrageous into the inhibitor binding cavity revealed that ligand is inserted in to "aromatic case" framed by LYS 231, PHE 232, VAL 224, LEU 223, ASP 195, GLY 83, LY 384, ARG 88, GLY 49, ALA 43, HIS 47 and the FAD aromatic ring of the protein. The binding is further stabilized by $\pi-\pi$ stacking and the oxygen of $\mathrm{NO}_{2}$ of the $\mathrm{N}$-aryl part of compound forms a hydrogen bond interaction with the OH of ALA 43 having value 3.063 (Figure 5).

In compound C-10, $1 \mathrm{WNY}$ PDB, Visual inspection of the pose of structure umbrageous into the inhibitor binding cavity revealed that ligand is inserted in to "aromatic case" framed by PRO 294, TYR 301, LYS 212, PRO 288, PHE 302, GLU 288, TYR 335, LYS 299 and the FAD aromatic ring of the protein (Figure 6) and 


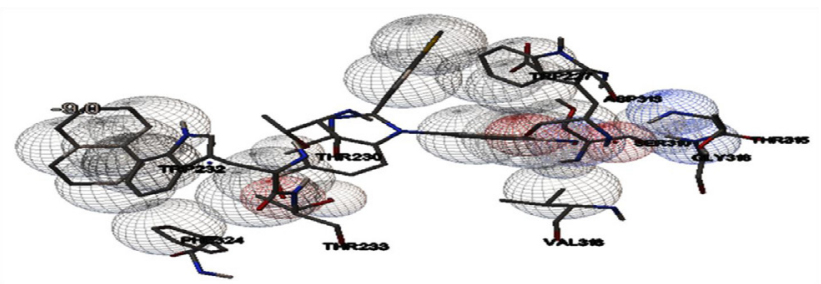

Figure 4: Docking Result of C-1 with PDB 1WNY.

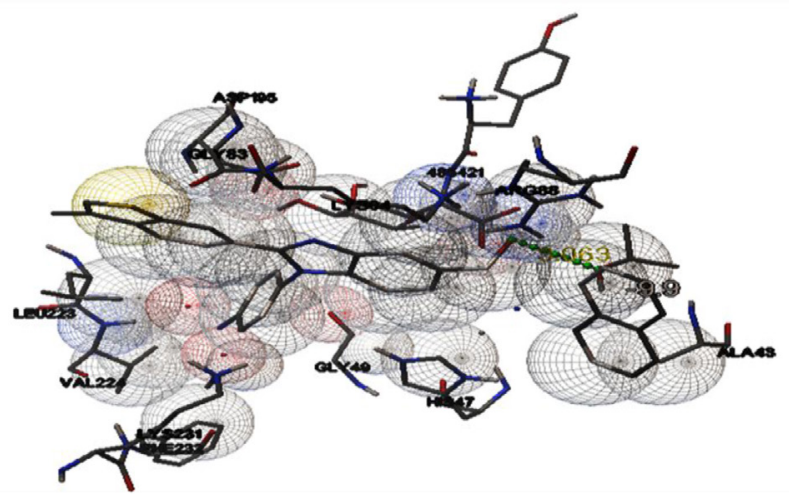

Figure 5: Docking Result of C-1 with PDB 1JIL.

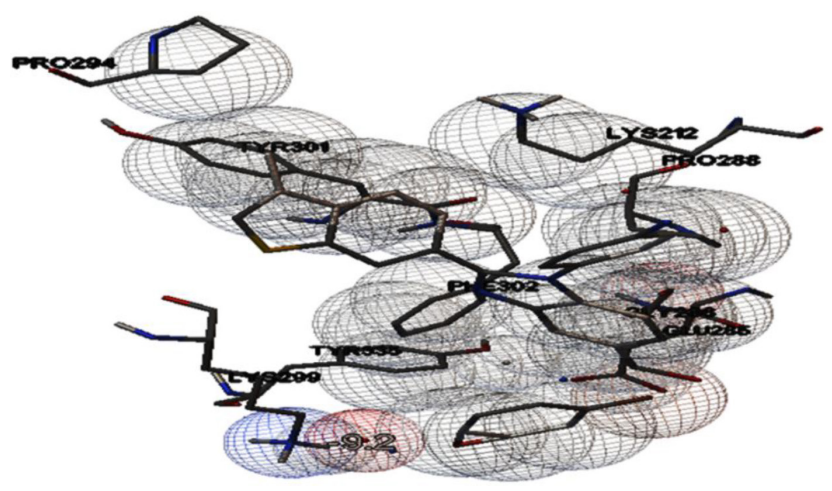

Figure 6: Docking Result of C-10 with PDB 1WNY.

1JIL PDB, Visual inspection of the pose of structure umbrageous into the inhibitor binding cavity revealed that ligand is inserted into "aromatic case" framed by THR 34, GLN 32, ARG 66, GLY 118, GLN 81, GLU 62, GLU 112, ARG 58, PHE 273 and the FAD aromatic ring of the protein (Figure 7).

In compound C-19, $1 \mathrm{WNY}$ PDB, Visual inspection of the pose of structure umbrageous into the inhibitor binding cavity revealed that ligand is inserted in to "aromatic case" framed by VAL 318, GLY 318, SER 310, THR 316, ASP 313, TRP 217, THR 229, TRF 232, PHE 324, GLY 325 and the FAD aromatic ring of the protein. The binding is further stabilized by $\pi-\pi$ stacking and the oxygen of $\mathrm{NO}_{2}$ of the $\mathrm{N}$-aryl part of compound forms a hydrogen bond interaction with

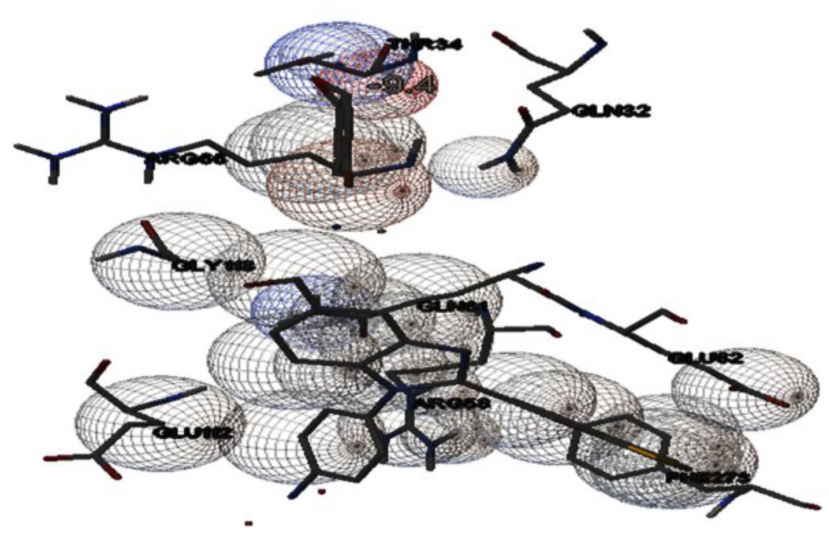

Figure 7: Docking Result of C-10 with PDB 1JIL.

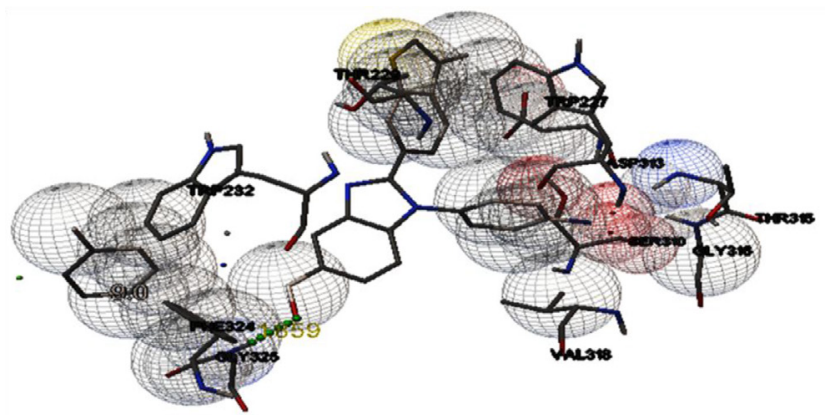

Figure 8: Docking Result of C-19 with PDB 1WNY.

the OH of GLY 325 having value 1.859 (Figure 8) and 1JIL PDB, Visual inspection of the pose of structure umbrageous into the inhibitor binding cavity revealed that ligand is inserted in to "aromatic case" framed by ALA 43, HIS 47, GLY 49, PHE 232, LYS 231, VAL 224, LEU 223, ASP 195, LYS 84, ARG 88 and the FAD aromatic ring of the protein. The binding is further stabilized by $\pi-\pi$ stacking and the oxygen of $\mathrm{NO}_{2}$ of the $\mathrm{N}$-aryl part of compound forms a hydrogen bond interaction with the protein having value 3.082 (Figure 9). Docking studies revealed that hydrogen bond interactions fix the ligands firmly and tightly in the active site.

\section{SAR (structure-activity relationship) studies}

1. Amide derivatives of 2-(3-methylbenzo[b]thiophen6-yl)-1-(4-nitrophenyl)-1H-benzo[d]imidazole5-carboxylic acid of good biological activity as compared with compound C.

2. Substiution of Bromo group (electron withdrawing group) at 2 and Chloro at 4 (electron withdrawing group) shows the best antimicrobial activity among the series i.e. compound C-10.

3. Substitution of naphthalene ring shows second best antimicrobial activity among the series i.e. compound C-1. 


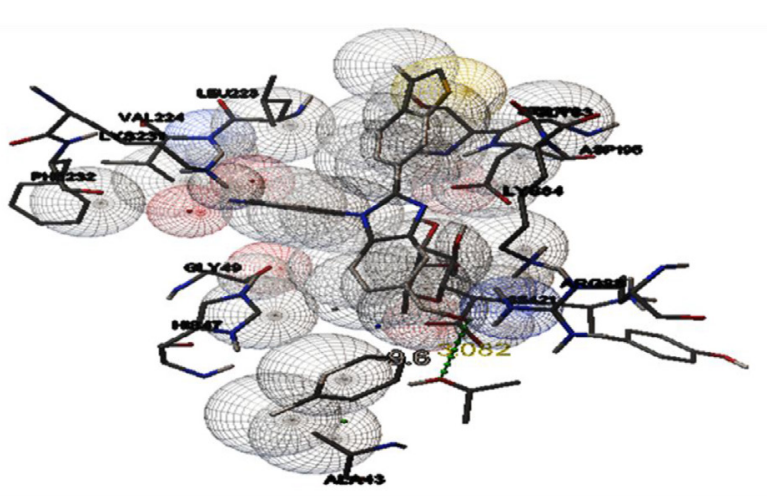

Figure 9: Docking Result of C-19 with PDB 1JIL.

4. Substiution of methyl group at 3 and Nitro at 4 shows the best antibacterial activity but decreases antifungal activity i.e. compound C-19.

5. Substiution of Nitro at 2(electron withdrawing group) and chloro(electron withdrawing group) at 4 shows the good antimicrobial activity i.e. compound C-23.

6. Di-nitro group (High electron withdrawing group) at 2,4 position shows poor antimicrobial activity among the series i.e. compound C-4.

7. Substiution of Chloro group at 2,5 shows the good antimicrobial activity i.e. compound C-5.

8. Substiution of di-floro at 2,3 and 2,6 shows the moderate antimicrobial activity i.e. compound C-2, C- 8 but substitution at 2,5 shows good activity i.e. compound C-6.

9. substitution of bromo at 2 and electron donating group at position at 4, vice versa shows good antimicrobial activity i.e. compound C-18 and C-9.

10. Substitution of one electron withdrawing group and one electron donating group at different position of aromatic ring shows moderate activity among the series i.e. compound C-11, C-18.

11. Substitution of one electron withdrawing group at different position of aromatic ring shows moderate activity i.e. compound C-13, C-14, C-16.

12. Antifungal activity decreases when aniline is replaced by phenylmethanamine shows week among the series i.e. compound C-22.

Antibacterial activity decreases when methyl group substituted at position 2,4 shows week among the series i.e. compound C-3.

\section{CONCLUSION}

In-vitro outcome exposed that a vast number of synthesized compounds were found to havetremendous antimicrobial activity. Among the library, C-1, C-10 and C-19 possessing admirable dock score by both software: AutoDock Vina/Schrodinger's maestro against 1wny.PDBQT:1 jil.PDBQT is -9.0:-9.9/ -5.144:5.328,-9.2:-9.4/ -5.007:-5.369, -9.0:-9.6/ -5.184:-5.264 and as compared to standard drugs dock score -10.2:10.6/-5.847:-5.895, -10.2:-10.6/-5.847:-5.895, -9.8:-9.9/ -6.323:-6.131.C-1, C-10 and C-19 derivatives also presented good MIC as compared to standard by tube dilution method. In- silico studies played an symbolic role in designing the potent ligands against aminoacyl-tRNA synthetase (AaRS) and tyrosyl-tRNA synthetase enzymes as well as interpreted/studyingthe binding pattern of designed/synthesized ligand within active pocket and excellent antimicrobial compounds can be used for in vivo studies for future. In-silico studies and in vitro results comply with each other.

\section{ACKNOWLEDGEMENT}

The authors are highly thankful to the Head, Department of Pharmaceutical Sciences and Natural Products, Central University of Punjab, Bathinda for providing essential facilities to accomplish this research study. The authors are also sincerely thankful to Dr. Vinod Devaraji Application Scientist Schrödinger LLC for his support to carry out the computational work.

\section{CONFLICT OF INTEREST}

The authors declare no conflict of interest.

\section{ABBREVIATIONS}

${ }^{13} \mathbf{C}$ NMR: Carbon 13 Nuclear magnetic resonance; ${ }^{1} \mathbf{H}$ NMR: Proton Nuclear magnetic resonance; 4-MBA: 4-Mercapto Benzoic acid; BOD: Biological oxygen demand; CFU: Colony forming unit; DCC: Di Cyclohexyl carbodimide; DCM: Dichloro methane; DMAP: 4-dimethyl amino pyridine; DMF: Dimethylsulfoxide; DMSO: Dimethyl sulfoxide; EDC: Ethylene dichloride; EDCI: 1-Ethyl 3-(3-dimethylaminopropyl) carbodiimide; HOBt: 1-Hydroxybenzotriazole; MIC: Minimum inhibitory concentrations; MS: Mass Spectra; MTCC: Microbial Type Culture Collection; MW: Molecular weight; OPLS: Optimized Potential for Liquid Simulations; PDB ID: Protein Data Bank Identification; PEG-5000: Poly Ethylene Glycol 5000; PPA: Polyphosphoric acid; RBF: Round Bottom Flask; RT: Room temperature; TEA: Triethylamine; TFA: Trifluro acetic acid; TLC: Thin layer chromatography; UV: Ultra violet. 


\section{REFERENCES}

1. Kulkarni KM, Jadhav SA, Patil PB, et al. Synthesis and antimicrobial screening of 2-mercaptobenzimidazole derivatives. Der Pharma Chemica. 2016;8(4):1-5

2. Qiu X, Janson CA, Smith WW, et al. Crystal structure of Staphylococcus aureus tyrosyl-tRNA synthetase in complex with a class of potent and specific inhibitors. Protein Science. 2001;10(10):2008-16.

3. Fukunaga R, Yokoyama S. Structural Basis for Substrate Recognition by the Editing Domain of Isoleucyl-tRNA Synthetase. J Mol Biol. 2006;359(4):901-12.

4. Soper TS, Manning JM. Different modes of action of inhibitors of bacterial d-amino acid transaminase: A target enzyme for the design of new antibacterial agents. J Biol Chem. 1981;256(9):4263-8.

5. Mehta P, Chovatiya P, Joshi SH. Synthesis, characterization and antimicrobial evaluation of some novel benzene sulfonylhydrazone derivatives of benzimidazole. Der Chemica Sinica. 2015;6(2):29-34.

6. Abdel AISH, Soliman FMA. Synthesis, some reactions, cytotoxic evaluation and antioxidant study of novel benzimidazole derivatives. Der Pharma Chemica. 2015;7(4):71-84.

7. Rao GM, Reddy YN, Kumar BV. Evaluation of analgesic and anti-inflammatory activities of $n$-mannich bases of substituted 2-mercapto-1 $h$-benzimidazoles. Int J Appl Biol Pharm. 2013;38-46.

8. Shingalapur RV, Hosamani KM, Keri RS, et al. Derivatives of benzimidazole pharmacophore: Synthesis, anticonvulsant, antidiabetic and DNA cleavage studies. Eur J Med Chem. 2010;45(5):1753-9.

9. Vaidyaa SD, Kumara BVS, Kumar RV, et al. Synthesis, Anti-bacterial, Anti-asthmatic and Anti-diabetic Activities of Novel N-Substituted-2(benzo[d]isoxazol-3-ylmethyl)1 H-benzimidazoles. J Heterocyclic Chem. 2007;44(3):685-91.

10. Tonelli M, Simone M, Tasso B, et al. Antiviral activity of benzimidazole derivatives. II. Antiviral activity of 2-phenylbenzimidazole derivatives. Bioorg Med Chem. 2010;18(8):2937-53.

11. Sharma MC, Kohli DV, Sharma S, et al. Synthesis and antihypertensive activity of 4'-\{2-[4-[2-(Substitutedphenyl)-4-oxo-thiazolidin-3-yl]-phenyl\} benzoimidazol-1-yl-methyl\}-biphenyl-2-carboxylic acids. Der Pharmacia Sinica. 2010;1(1):58-73.

12. Cui J, Qi B, Gan C, et al. Synthesis and in vitro antiproliferative evaluation of some b-norcholesteryl benzimidazole and benzothiazole derivatives. Mar Drugs. 2015;13(4):2488-504.
13. Revathi R, Perumal RV, Pai KSR, et al. Design, development, drug-likeness and molecular docking studies of novel piperidin-4-imine derivatives as antitubercular agents. Drug Des Dev Ther. 2015;9:3779-87.

14. Sivakumar R, Pradeepchandran R, Jayaveera KN, et al. Benzimidazole: An Attractive Pharmacophore in Medicinal Chemistry. IJPR. 2011;3(3):19-31.

15. Salahuddin, Shaharyar BM, Mazumder A. Benzimidazoles: A biologically active compounds. Arabian Journal of Chemistry. 2017;10:S157-73.

16. Shen $\mathrm{J}, \mathrm{Xu} \mathrm{X}$, Cheng $\mathrm{F}$, et al. Virtual screening on natural products for discovering active compounds and target information. Current Medicinal Chemistry. 2003;10(21):2327-42.

17. Rizvi SMD, Shakil S, Haneef M. A simple click by click protocol to perform docking: Autodock 4.2 made easy for non-bioinformaticians. Excli J. 2013;12:831-57.

18. Maestro, version 10.2, Schrödinger, LLC, New York, NY. 2015

19. Glide, Version 6.6, Schrödinger, LLC, New York, NY. 2015.

20. Rao NR. Molecular Modelling: A powerful tool for Drug Design and Molecular Docking. Resonance. 2004;9(5):51-60.

21. Kini D, Kumar H, Ghate M. Microwave Assisted Liquid Phase Synthesis of Benzimidazolo Benzothiophenes for Antimicrobial Activity. Eur J Chem. 2009;6(S1):S25-32.

22. Pan PC, Sun CM. Liquid phase synthesis of arylamines and its application to the benzimidazolone via nucleophilic aryl substitution. Bioorg Med Chem Lett. 1999;9(11):1537-40.

23. Urban PL. Quantitative mass spectrometry: An overview. Phil Trans R Soc A. 2017;374:20150382.

24. Williams K. PNMR spectroscopy. Organic Spectroscopy. Macmillan Publishing Co. Inc. 1991;127-30.

25. Stahl E, Ashworth MRF. Thin layer Chromatography. A Laboratory Handbook. Springer Verlag. $2^{\text {nd }}$ ed. Berlin, Heidelberg, Newyork. 1996;6-29.

26. Chan LC, Cox BG. Kinetics of Amide Formation through Carbodiimide/ N-Hydroxybenzotriazole (HOBt) Couplings. J Org Chem. 2007;72(23):8863-9.

27. Cappucino JG, Sherman N. Microbiology-A laboratory manual. Addison Wesley, California.1999;263.

28. Kowser MM, Fatema N. Determination of MIC and MBC of selected azithromycin capsule commercially available in Bangladesh. The Orion Med J. 2009;32(1):619-20.

29. Indian Pharmacopoeia. Indian Pharmacopoeia Commission, Ghaziabad, India. 2010;27-8.

30. Godschalk F, Genheden S, Söderhjelm P, et al. Comparison of MM/GBSA calculations based on explicit and implicit solvent simulations. Phys Chem Chem Phy. 2013;15(20):7731-9.

\section{SUMMARY}

There has been a repetitive fight among humans and the multitude of micro-organisms that origin infection and disease. Infectious diseases are accountable for large figure of deaths in the world population. The casual use and overconsumption of antibiotics has led to the emergence of antibiotic-resistant strains. Therefore, there is a vital need for the growth of new antimicrobial drugs. In the study, we have synthesized and investigated antimicrobial activities of novel derivatives with molecular docking studies. In this, amide derivatives of 2-(3-methylbenzo[b]thiophen-6-yl)-1-(4-nitrophenyl)-1H-benzo[d]imidazole-5-carboxylic acid were synthesized by focusing the aminoacyl-tRNA synthetase (AaRS) and tyrosyl-tRNA synthetase ensymes and inhibition by docking study precedence to antimicrobial action. Studies were accomplished on a designed library of amide derivatives with the help of docking softwares i.e. AutoDock Vina 4.2 and Schrodinger's maestro package against crystal structure of enzymes (PDB ID: 1wny.PDBQT and 1jil.PDBQT). Based upon their dock score, superlative 23 focused amide derivatives of 2-(3-methylbenzo[b]thiophen-6-yl)-1-(4-nitrophenyl)$1 \mathrm{H}$-benzo[d]imidazole-5-carboxylic acid were synthesized and further investigated for in vitro antimicrobial. In-silico studies and in vitro results comply with each other. 


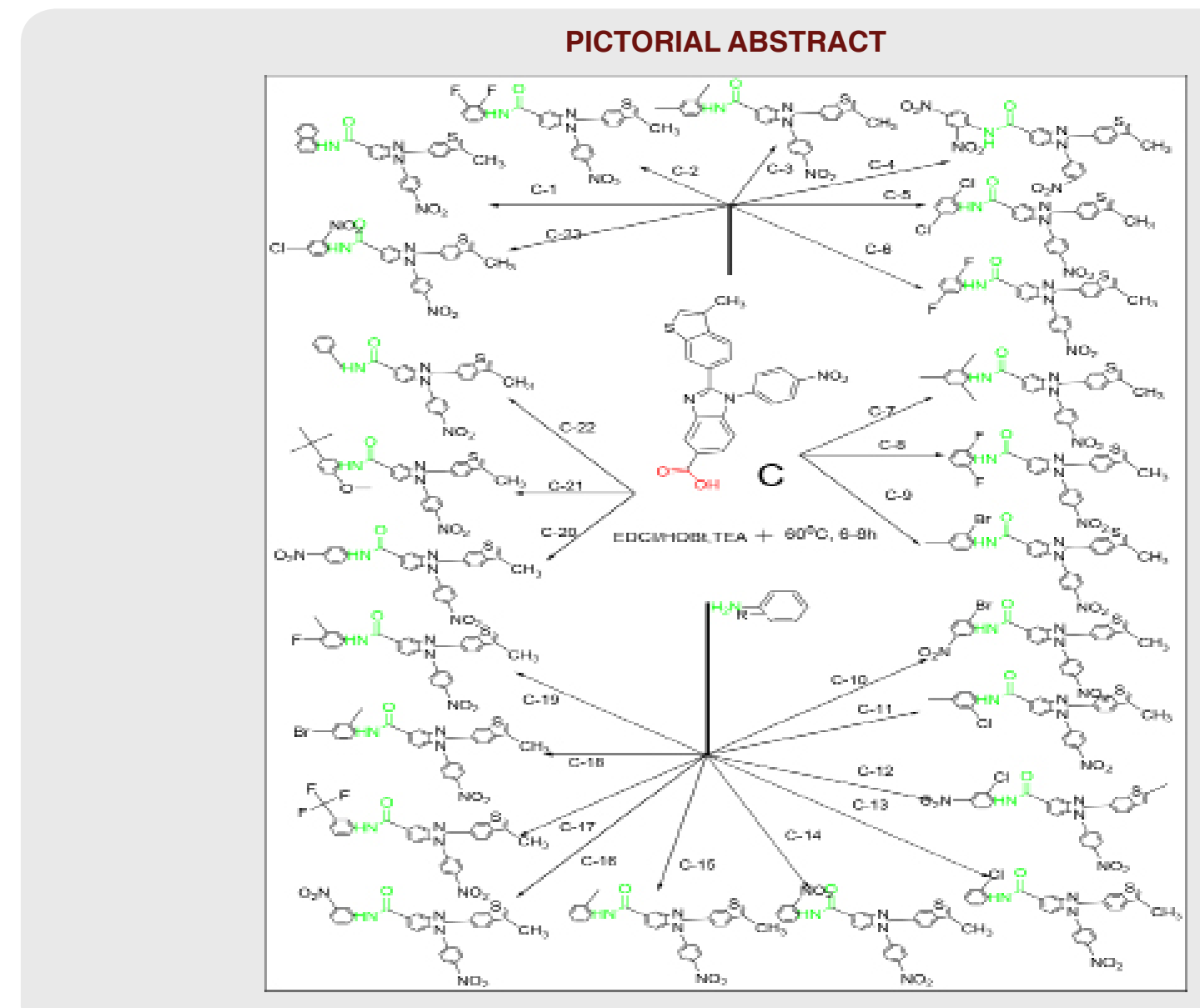

Cite this article: Hooda T, Sharma S, Goyal N. Synthesis, in-silico Designing, SAR and Microbiological Evaluation of Novel Amide Derivatives of 1-(4-Nitrophenyl)-2-(3-methylbenzo[b]thiophen-6-yl)-1H-benzo[d]imidazole-5-carboxylic Acid. Indian J of Pharmaceutical Education and Research. 2020;54(2):471-83. 